\title{
Planck-Scale Mass Equidistribution of Toral Laplace Eigenfunctions
}

\author{
Andrew Granville ${ }^{1,2}$, Igor Wigman ${ }^{3}$ (D) \\ 1 Département de mathématiques et de statistique, Université de Montréal, CP 6128 succ. Centre-Ville, \\ Montreal, QC H3C 3J7, Canada. E-mail: and.granville@gmail.com \\ 2 Department of Mathematics, University College London, Gower Street, London WC1E 6BT, England \\ 3 Department of Mathematics, King's College London, Strand, London WC2R 2LS, England, UK. \\ E-mail: igor.wigman@kcl.ac.uk
}

Received: 27 December 2016 / Accepted: 15 May 2017

Published online: 10 July 2017 - (C) The Author(s) 2017. This article is an open access publication

\section{Dedicated to the memory of Javier Cilleruelo}

\begin{abstract}
We study the small scale distribution of the $L^{2}$-mass of eigenfunctions of the Laplacian on the two-dimensional flat torus. Given an orthonormal basis of eigenfunctions, Lester and Rudnick (Commun. Math. Phys. 350(1):279-300, 2017) showed the existence of a density one subsequence whose $L^{2}$-mass equidistributes more-or-less down to the Planck scale. We give a more precise version of their result showing equidistribution holds down to a small power of log above Planck scale, and also showing that the $L^{2}$-mass fails to equidistribute at a slightly smaller power of log above the Planck scale. This article rests on a number of results about the proximity of lattice points on circles, much of it based on foundational work of Javier Cilleruelo.
\end{abstract}

\section{Introduction}

1.1. Background and motivation. Let $\mathcal{M}$ be a smooth, compact, $d$-dimensional Riemann manifold, and with no loss of generality we assume that $\operatorname{Vol}(\mathcal{M})=1$. We are interested in the Laplace spectrum of $\mathcal{M}$ (also called "energy levels"): these are the eigenvalues, $E$, of the equation

$$
\Delta f+E f=0 \text {. }
$$

It is well-known that the eigenvalue spectrum $\left\{E_{j}\right\}_{j \geq 1}$ is discrete, that $E_{j} \rightarrow \infty$, and we let $\phi_{j}$ be a corresponding orthonormal basis of eigenfunctions. Shnirelman's Theorem $[7,16,18]$ asserts that if $\mathcal{M}$ is chaotic (that is, the geodesic flow on $\mathcal{M}$ is ergodic), then there is a subsequence $\left\{E_{j_{k}}\right\}_{j \geq k}$ of $\left\{E_{j}\right\}_{j \geq 1}$, of density one, for which the $\phi_{j}$ are $L^{2}$-equidistributed in the phase-space; in particular, for every "nice" domain on the configuration space $\mathcal{A} \subseteq \mathcal{M}$ we have

$$
\frac{\int_{\mathcal{A}} \phi_{j_{k}}^{2}(y) d y}{\operatorname{Vol}(\mathcal{A})} \rightarrow 1,
$$


where $\operatorname{Vol}(\mathcal{A})$ is the ( $d$-dimensional) volume of $\mathcal{A}$.

Berry's widely believed conjecture [1,2] goes beyond Shnirelman's Theorem, asserting that if $\mathcal{M}$ is chaotic then (1) holds for any $\mathcal{A}=\mathcal{A}_{j_{k}}$ which shrinks slightly slower than the Planck scale $E_{j_{k}}^{-1 / 2}$. More precisely, let

$$
B_{x}(r) \subseteq \mathcal{M}
$$

be the radius $r>0$ geodesic ball centred at $x$. Then there should exist a density 1 subsequence $\left\{\phi_{j_{k}}\right\}_{k \geq 1}$ of energy levels, such that (1) holds uniformly for all $x \in \mathcal{M}$, $r>r_{0}\left(E_{j_{k}}\right)$ with $B_{x}(r)$ in place of $A$, as long as

$$
\lim _{E \rightarrow \infty} r_{0}(E) \cdot E^{1 / 2}=\infty
$$

i.e.

$$
\sup _{\substack{r>r_{0}(E) \\ x \in \mathcal{M}}}\left|\frac{\int B_{x}(r)}{\operatorname{Vol}\left(B_{x}(r)\right)}-1\right| \rightarrow 0 .
$$

There are only a few such results in the literature with $r$ small:

- Luo and Sarnak [15] showed this for $r>E^{-\alpha}$ for some small $\alpha>0$, for the modular surface, where the eigenfunctions are the eigenfunctions of all Hecke operators, and Young [17] showed this for all eigenfunctions for $r>E^{-1 / 4+o(1)}$, assuming the Generalized Riemann Hypothesis;

- Hezari and Rivière [11] and Han [9] showed the integral is the expected value up to a multiplicative constant, for $r>(\log E)^{-\alpha}$ for some small $\alpha>0$, on negatively curved manifolds. Han [10] also showed this for "symmetric" manifolds (i.e., manifolds on which the group of isometries act transitively) on which the lower bound on $r$ depends on the growth rate of the eigenspace dimensions (the "spectral degeneracy").

- Small scale mass equidistribution of toral eigenfunctions (on $\mathbb{T}^{d}=(\mathbb{R} / \mathbb{Z})^{d}$ ) was studied for the first time by Hezari and Rivière [12, Corollary 1.5], who proved that for "most" of the eigenfunctions the left-hand side of (3) is bounded on shrinking balls of radius $r=E^{-1 /(7 d+4)}$. This result was improved to equidistribution (3) holding uniformly for all $r>E^{-1 / 2(d-1)+o(1)}$ in the important paper of Lester and Rudnick [14] and, therein, this exponent was shown to be "best possible" by Bourgain [14, Theorem 4.1].

1.2. Toral eigenfunctions. Our starting point is the work of Lester and Rudnick [14, Theorem 1.1], who considered the small-scale equidistribution (3) of Laplace eigenfunctions on the (completely integrable) $d$-dimensional torus $\mathbb{T}^{d}=(\mathbb{R} / \mathbb{Z})^{d}$ (but which does not satisfy the chaotic condition from Berry's conjecture). For $d=2$ they proved that if $\left\{\phi_{j}\right\}$ is an orthonormal basis of $L^{2}\left(\mathbb{T}^{2}\right)$, then there exists a density one subsequence $\left\{j_{k}\right\}$ of the positive integers, for which (3) holds provided that $r_{0}>E^{-1 / 2+o(1)}$, which is close to the full (optimal) "Planck range" (2); we prove a strong version of this result below (see Theorem 1.2).

Let

$$
S=\left\{a^{2}+b^{2}: a, b \in \mathbb{Z}\right\}
$$


be the set of all integers expressible as the sum of two squares. For $n \in S$ let $\mathcal{E}_{n}$ be the set of lattice points lying on the circle of radius $\sqrt{n}$, namely

$$
\mathcal{E}_{n}=\left\{\lambda \in \mathbb{Z}^{2}:\|\lambda\|^{2}=n\right\}
$$

which has size $\# \mathcal{E}_{n}=r_{2}(n)$, the number of different ways of expressing $n$ as the sum of two squares.

The eigenvalues of the Laplacian of $\mathbb{T}^{2}$ are the numbers $E=4 \pi^{2} n$ with ${ }^{1} n \in S$, and the corresponding space of (complex-valued) eigenfunctions is

$$
f_{n}(x)=\sum_{\lambda \in \mathcal{E}_{n}} c_{\lambda} e(\langle x, \lambda\rangle)
$$

of dimension $r_{2}(n)$. We will further assume that the $f_{n}$ are real-valued, so that

$$
c_{-\lambda}=\overline{c_{\lambda}}
$$

and multiply through by a constant so that

$$
\left\|f_{n}\right\|_{2}^{2}=\sum_{\lambda \in \mathcal{E}_{n}}\left|c_{\lambda}\right|^{2}=1
$$

Landau $[4,13, \S 1.8]$ proved that

$$
|\{n \in S: n \leq N\}| \sim \kappa_{L R} \cdot \frac{N}{\sqrt{\log N}}\left(1+O\left(\frac{1}{\log N}\right)\right),
$$

where $\kappa_{L R}:=\frac{\pi}{4} \kappa^{\prime}=0.76422 \ldots$ with

$$
\kappa^{\prime}:=\prod_{p \equiv 1}\left(1-1 / p^{2}\right)^{1 / 2} .
$$

Ramanujan rediscovered this, and observed that

$$
r_{2}(n)=O_{\epsilon}\left(n^{\epsilon}\right) \text { for every } \epsilon>0
$$

\subsection{Planck-scale mass equidistribution for Bourgain-Rudnick sequences.}

Definition 1.1. For $\delta>0$ we say that a sequence $\{n\} \subseteq S$ satisfies the Bourgain-Rudnick condition, denoted by $B R(\delta)$, if there exists $C>0$ such that

$$
\min _{\substack{\lambda, \lambda^{\prime} \in \mathcal{E}_{n} \\ \lambda \neq \lambda^{\prime}}}\left\|\lambda-\lambda^{\prime}\right\|>C \cdot n^{1 / 2-\delta}
$$

\footnotetext{
${ }^{1}$ By an abuse of notation, $n$ is commonly referred to as an "energy level" rather than the corresponding $E=4 \pi^{2} n$.
} 
Bourgain-Rudnick [3, Lemma 5] proved that for every $C>0$,

$$
B(N ; \delta):=\#\left\{n \leq N, n \in S: \min _{\substack{\lambda, \lambda^{\prime} \in \mathcal{E}_{n} \\ \lambda \neq \lambda^{\prime}}}\left\|\lambda-\lambda^{\prime}\right\| \leq C \cdot n^{1 / 2-\delta}\right\} \ll N^{1-\delta / 3} .
$$

(The $B(N ; \delta)$ is also implicitly dependent on $C$.) Together with Landau's estimate (7), this implies that a generic sequence $\{n\} \subseteq S$ satisfies the $B R(\delta)$ condition, for arbitrary $\delta>0$. Theorem 1.4 below allows us to improve this bound to

$$
B(N ; \delta) \ll N^{1-\delta}(\log N)^{1 / 2},
$$

which is perhaps close to the true number of exceptional $n$. For all eigenfunctions corresponding to energy levels satisfying the $B R(\delta)$ condition we prove the following uniform equidistribution result with a strong upper bound on the discrepancy, for close to the full Planck range:

Theorem 1.2. Let $\epsilon>\delta>0$ and $0<\eta<\epsilon-\delta$. For all sufficiently large $n$ satisfying Bourgain-Rudnick's $B R(\delta)$ condition and all $f$ for which $\|f\|=1$ we have

$$
\sup _{x \in \mathbb{T}, r>n^{-1 / 2+\epsilon}}\left|\frac{\int_{B_{x}(r)} f(y)^{2} d y}{\pi r^{2}}-1\right| \ll n^{-3 \eta / 2} .
$$

Theorem 1.2 implies Lester and Rudnick's result [14] for 2-dimensional tori (mentioned at the beginning of section 1.2), as (11) is so much smaller than (7).

1.4. On the number of exceptional energy levels. Our goal is to estimate the number of exceptions to $B R(\delta)$, for given $\delta>0$. To do this we obtain a precise estimate for

$$
B^{*}(N ; \delta):=\#\left\{\left(\lambda, \lambda^{\prime}\right):\|\lambda\|^{2}=\left\|\lambda^{\prime}\right\|^{2} \leq N, 0<\left\|\lambda-\lambda^{\prime}\right\| \leq C\|\lambda\|^{1-2 \delta}\right\},
$$

which yields a better bound than (11) for the number of exceptions to $B R(\delta)$, as $B(N ; \delta) \leq B^{*}(N ; \delta)$.

Theorem 1.3. Fix $0<\delta<\frac{1}{2}$, and a constant $C>0$. Then

$$
B^{*}(N ; \delta)=\frac{4 C}{\pi} \cdot \frac{1-2 \delta}{1-\delta} \cdot N^{1-\delta} \log N\left(1+O\left(\frac{1}{\sqrt{\log N}}\right)\right) .
$$

The proof of Theorem 1.3, given in section 6.1, is a relatively straightforward application of a more general Theorem 6.1. Theorem 1.3 implies in particular the upper bound

$$
B(N ; \delta) \ll N^{1-\delta} \log N
$$

for the number of $n \in S$ not satisfying $B R(\delta)$, but not a lower bound. This is because Theorem 1.3 evaluates the number of close-by pairs of lattice points rather than the corresponding radii, which a priori can result in substantial over-counting in $B(N ; \delta)$ as a particular radius might correspond to many different pairs. That, in fact, this is so, follows from the following theorem; it implies that the average number of close-by pairs 
corresponding to radii not satisfying $B R(\delta)$ is growing to infinity (cf. (17) vs. (14)). We define

$$
\mathcal{G}^{*}(N ; M):=\#\left\{n \leq N: \exists \lambda, \lambda^{\prime} \in \mathcal{E}_{n} .0<\left\|\lambda-\lambda^{\prime}\right\|<M\right\}
$$

Theorem 1.4. Let $M=M(N)$ be a function of $N$.

(1) Under the assumption

$$
(\log N)^{3} \leq M \leq \frac{N^{1 / 2}}{(\log N)^{21}},
$$

$\mathcal{G}^{*}(N ; M)$ satisfies the asymptotic law

$$
\mathcal{G}^{*}(N ; M)=\frac{2 \kappa^{\prime}}{\pi} \cdot \sqrt{N} M\left((2 \log M)^{1 / 2}+O(1)\right),
$$

where $\kappa^{\prime}$ is as in (8).

(2) With no assumption on $M$ we have the upper bound

$$
\mathcal{G}^{*}(N ; M) \leq \frac{2 \kappa^{\prime}}{\pi} \cdot \sqrt{N} M\left((2 \log M)^{1 / 2}+O(1)\right)
$$

The proof of Theorem 1.4, given in section 6.3, is a straightforward application of the more general Theorem 6.2 below. It also yields the aforementioned upper bound

$$
B(N ; \delta) \ll N^{1-\delta}(\log N)^{1 / 2}
$$

for the number of $n \in S$ not satisfying $B R(\delta)$, stronger than (14) above. Comparing the second part of Theorem 1.4 to (7) we see that if

$$
\psi(n)=o\left(n^{1 / 2} /(\log n)\right),
$$

then, for almost all $n$, we have $\left\|\lambda-\lambda^{\prime}\right\|>\psi(n)$ whenever

$$
\|\lambda\|^{2}=\left\|\lambda^{\prime}\right\|^{2}=n
$$

with $\lambda \neq \lambda^{\prime}$. (Therefore $B R\left((1+\epsilon) \frac{\log \log n}{\log n}\right)$ holds for almost all $n$.)

1.5. Planck-scale equidistribution for flat functions, valid for arbitrary energies. Without ruling out the possible existence of close-by pairs of lattice points we will not be able to prove a uniform result for all energy levels, though we do get fairly precise results in terms of

$$
\min _{\lambda \neq \lambda^{\prime} \in \mathcal{E}_{n}}\left\|\lambda-\lambda^{\prime}\right\|
$$

In Corollary 2.2 we show that Berry's conjecture is generically true for all

$$
r \geq r_{2}(n)^{2 / 3}(\log n)^{\epsilon} / \min _{\lambda \neq \lambda^{\prime}}\left\|\lambda-\lambda^{\prime}\right\|
$$


while in Proposition 3.1 we show that Berry's conjecture is generically false for some

$$
r \gg 1 / \min _{\lambda \neq \lambda^{\prime}}\left\|\lambda-\lambda^{\prime}\right\|
$$

There is not much difference in these two bounds as $r_{2}(n)$ is bounded by a small power of $\log n$, for almost all $n$.

We might instead ask for the typical error term when considering the ball centre $x \in \mathbb{T}$ as random, uniformly distributed on the torus. By evaluating the variance of the corresponding variable we will be able to infer that the $L^{2}$ mass is equidistributed for most $x$ (see Corollaries 1.7 and 1.10 below).

For $f=f_{n}$ of the form (4) and $r>0$ we define

$$
X=X_{f, r}=X_{f, r ; x}=X_{x}=\int_{B_{x}(r)} f(y)^{2} d y,
$$

thinking of $X$ as a random variable with the ball centre $x \in \mathbb{T}$ drawn at random, uniformly, on the torus. The expectation

$$
\mathbb{E}[X]=\int_{\mathbb{T}} X_{f, r ; x} d x,
$$

of the $L^{2}$ mass is simply the area of $B_{x}(r)$, as $\|f\|=1$. Therefore:

Lemma 1.5. For every $r>0$ we have

$$
\mathbb{E}[X]=\pi r^{2} .
$$

The corresponding variance is defined as

$$
\mathcal{V}(X)=\int_{\mathbb{T}} X_{f, r ; x}^{2} d x-\mathbb{E}[X]^{2}=\int_{\mathbb{T}}\left(\int_{B_{x}(r)} f(y)^{2} d y-\pi r^{2}\right)^{2} d x .
$$

The following result implies that the $L^{2}$-mass of any "flat" $f$ is equidistributed on "most" of the balls, i.e.,

$$
\mathcal{V}(X)=o\left(r^{4}\right)=o\left(\mathbb{E}[X]^{2}\right)
$$

(see Corollaries 1.7 and 1.10).

Theorem 1.6. For every $n \in S, f=f_{n}$ a function of the form (4), satisfying (6), and any small $\xi=\xi(n)>0$, we have the following bound on the variance (19)

$$
\mathcal{V}(X) \ll\left(\sum_{0<\left\|\lambda-\lambda^{\prime}\right\|<1 /(\xi r)}\left|c_{\lambda} c_{\lambda^{\prime}}\right|^{2}+\xi^{3}\right) \cdot r^{4},
$$

where the constant in the '«'-notation is absolute. 
Note that if $\mathcal{V}(X) \ll \delta^{4} r^{4}$ then the measure of the set

$$
\mathcal{X}=\mathcal{X}\left(f_{n}, r ; \delta\right)=\left\{x \in \mathbb{T}:\left|\int_{B_{x}(r)} f(y)^{2} d y-\pi r^{2}\right|>\delta r^{2}\right\}
$$

of centres $x \in \mathbb{T}$ violating equidistribution, is $\ll \delta^{2}$. The following corollary shows that if the "weights" of the coefficients $c_{\lambda}$ are smoothly distributed around the circle of radius $\sqrt{n}$, then $\mathcal{V}(X)=o\left(r^{4}\right)$. To formulate it we will need the notation $\eta$ as follows. Suppose that for some $\epsilon$ we have $\left|c_{\lambda}\right|^{2} \leq \epsilon$ for every $\lambda \in \mathcal{E}_{n}$. Let

$$
\eta=\eta\left(\left\{c_{\lambda}\right\}_{\lambda \in \mathcal{E}_{n}} ; \epsilon\right)>0
$$

be the maximal possible number such that for all $\alpha \in \mathbb{C}$ with $|\alpha|=\sqrt{n}$ we have

$$
\sum_{\lambda:\|\lambda-\alpha\|<\eta \sqrt{n}}\left|c_{\lambda}\right|^{2} \leq \epsilon
$$

that such $\eta$ exists follows from that fact that (21) is satisfied for all

$$
\eta<\frac{1}{\sqrt{2} \sqrt{n}} .
$$

One expects that for "most" functions $f_{n}$ in (4) satisfying (6), the inequality (21) is satisfied with $\eta \gg_{\epsilon} 1$ sufficiently small.

Corollary 1.7. Fix $\epsilon>0$ and assume that for each $\lambda \in \mathcal{E}_{n}$ we have $\left|c_{\lambda}\right|^{2} \leq \epsilon$. Then for all $r \geq 1 /(\epsilon \eta \sqrt{n})$ with $\eta$ as in (21), we have

$$
\mathcal{V}(X) \ll \epsilon r^{4},
$$

where the constant in the '«'-notation is absolute.

That the assumptions of Corollary 1.7 are "usually" satisfied (i.e., that the weight of the coefficients $c_{\lambda}$ are smoothly distributed for "most" $f$ ) is supported by the following result which shows that the lattice points on the circle of radius $\sqrt{n}$ are not overly crowded together:

Theorem 1.8. Fix $\epsilon>0$ sufficiently small. For every integer $n \in S$ with $r_{2}(n)>0$ we have

$$
\#\left\{\alpha, \beta \in \mathcal{E}_{n}:|\alpha-\beta| \leq n^{1 / 2-\epsilon}\right\} \ll_{\epsilon} r_{2}(n)^{2-\epsilon} .
$$

One can show that if

$$
\epsilon_{n}:=\frac{\log \log r_{2}(n)}{\log r_{2}(n)}
$$

the bound is

$$
\#\left\{\alpha, \beta \in \mathcal{E}_{n}:|\alpha-\beta| \leq n^{1 / 2-\epsilon_{n}}\right\} \ll \frac{r_{2}(n)^{2}}{\log r_{2}(n)}
$$


The proof yields that the upper bound in Theorem 1.8 may be improved to

$$
\#\left\{\alpha, \beta \in \mathcal{E}_{n}:|\alpha-\beta| \leq n^{1 / 2-\epsilon}\right\} \ll_{\epsilon} r_{2}(n)^{2-\tau \epsilon}
$$

for any fixed $\tau<4$.

Theorem 1.8 suggests that for all "reasonable" choice of coefficients $c_{\lambda}$ the r.h.s. of (20) is $o\left(r^{4}\right)$. Here we propose a possible notion of "reasonable".

Definition 1.9. (Flat and ultraflat functions).

(1) Let $\left\{f_{n}\right\}_{n \in S}$ be a sequence of functions as in (4), and

$$
\epsilon_{n}:=\frac{\log \log r_{2}(n)}{\log r_{2}(n)}
$$

We say that $\left\{f_{n}\right\}$ is flat if

$$
\max _{\substack{\alpha \in \mathbb{C} \\|\alpha|=\sqrt{n}}} \sum_{\lambda \in \mathcal{E}_{n}:\|\lambda-\alpha\|<n^{1 / 2-\epsilon_{n}}}\left|c_{\lambda}\right|^{2}=o_{n \rightarrow \infty} \text { (1). }
$$

(2) Let $f=f_{n}$ be a function as in (4). For $\epsilon>0$ we say that $f$ is $\epsilon$-ultraflat if for every $\lambda \in \mathcal{E}_{n}$,

$$
\left|c_{\lambda}\right|^{2} \leq \frac{1}{r_{2}(n)^{1-\epsilon}}
$$

Corollary 1.10. (1) For all $\left\{f_{n}\right\}_{n \in S}$ flat with $r \gg 1 / n^{1 / 2-2 \epsilon_{n}}$, where $\epsilon_{n}$ is given by (24), we have

$$
\mathcal{V}(X)=o\left(r^{4}\right)
$$

(2) If $f$ is $\epsilon$-ultraflat then for all $r \gg 1 / n^{1 / 2-4 \epsilon}$ we have

$$
\mathcal{V}(X) \ll r_{2}(n)^{-\epsilon} r^{4}
$$

1.6. Outline of the paper. In section 2, we give a proof of Theorem 1.2. In section 3 we construct a counterpoint to Theorem 1.2: a sequence of eigenfunctions corresponding to a density one sequence of energy levels, and balls with radii satisfying (2) that do not possess a "fair" share of the $L^{2}$-mass (see Corollary 3.2 and also Remark 3.3). Section 4 is dedicated to the proofs of Theorem 1.6, and Corollaries 1.7 and 1.10. The proof of Theorem 1.8 is given towards the end of section 5, after some considerable preparatory work. The proofs of theorems 1.3 and 1.4 will be given in sections 6.1 and 6.3 respectively; these are straightforward applications of the more general theorems 6.1 and 6.2 respectively. 


\section{Proof of Theorem 1.2}

The following lemma gives an exact formula for the error term and so will be useful in the proof of Theorem 1.2, and beyond.

Lemma 2.1. Let $f_{n}$ be given by (4) with (6) satisfied, $x \in \mathbb{T}$ and $r>0$. We have the identity

$$
\int_{B_{x}(r)} f_{n}^{2} d y-\pi r^{2}=2 \pi r^{2} \sum_{\lambda \neq \lambda^{\prime}} c_{\lambda} \overline{c_{\lambda}^{\prime}} e\left(\left\langle x, \lambda-\lambda^{\prime}\right\rangle\right) \frac{J_{1}\left(r\left\|\lambda-\lambda^{\prime}\right\|\right)}{r\left\|\lambda-\lambda^{\prime}\right\|},
$$

where $J_{1}$ is the Bessel function of the first kind.

We note that $J_{1}(t)$ oscillates between positive and negative values, and that for all $T>0$,

$$
\max _{T \leq t<2 T}\left|J_{1}(t)\right| \asymp \min \left\{T, \frac{1}{T^{1 / 2}}\right\} .
$$

Before giving a proof of Lemma 2.1 we formulate the following corollary establishing an explicit relation between the closest pairs of lattice points and radii satisfying the equidistribution (3), of independent interest, towards proving Theorem 1.2.

Corollary 2.2. Given $f_{n} \in \mathcal{E}_{n}$ as in (4), satisfying (6), $x \in \mathbb{T}, P \geq 1$ sufficiently large, and

$$
r \geq H \cdot \frac{r_{2}(n)^{2 / 3}}{\min _{\lambda \neq \lambda^{\prime}}\left\|\lambda-\lambda^{\prime}\right\|}
$$

we have

$$
\int_{B_{x}(r)} f_{n}^{2} d y=\left\{\pi+O\left(\frac{1}{H^{3 / 2}}\right)\right\} r^{2}
$$

where the constant involved in the ' $O$ '-notation is absolute.

Corollary 2.2 yields that for $H=H(n) \rightarrow \infty$ we have

$$
\int_{B_{x}(r)} f_{n}^{2} d y=\{\pi+o(1)\} r^{2},
$$

uniformly for all $r$ satisfying (28).

Proof of Corollary 2.2 assuming Lemma 2.1. Let

$$
R=r \cdot \min _{\lambda \neq \lambda^{\prime}}\left\|\lambda-\lambda^{\prime}\right\|
$$

so that $(28)$ is

$$
R \geq H \cdot r_{2}(n)^{2 / 3}
$$


Lemma 2.1 together with (27) then yield

$$
\left|\int_{B_{x}(r)} f_{n}^{2} d y-\pi r^{2}\right| \ll r^{2} \cdot R^{-3 / 2}\left(\sum_{\lambda}\left|c_{\lambda}\right|\right)^{2} .
$$

But

$$
\left(\sum_{\lambda}\left|c_{\lambda}\right|\right)^{2} \leq \sum_{\lambda} 1 \cdot \sum_{\lambda}\left|c_{\lambda}\right|^{2}=r_{2}(n)
$$

by (6) and the Cauchy-Schwarz inequality, so the upper bound in (31) is

$$
\left|\int_{B_{x}(r)} f_{n}^{2} d y-\pi r^{2}\right| \ll r^{2} \cdot \frac{r_{2}(n)}{R^{3 / 2}} \ll \frac{r^{2}}{H^{3 / 2}}
$$

by (30). The latter inequality is precisely the statement (29) of Corollary 2.2.

Proof of Theorem 1.2. Since we assumed the $B R(\delta)$ condition (10), an application of Corollary 2.2 with $H=n^{\eta}$ yields that

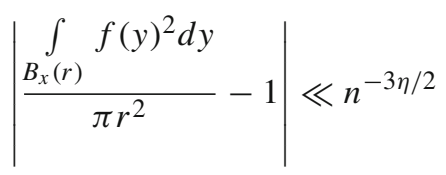

holds uniformly for all

$$
r>n^{-1 / 2+(\delta+\eta)} \cdot r_{2}(n)^{2 / 3} .
$$

That (32), in particular, holds for all $r>n^{-1 / 2+\epsilon}$, as claimed (12) in Theorem 1.2, follows from (9) and our assumption $0<\eta<\epsilon-\delta$.

Now we finally prove Lemma 2.1.

Proof of Lemma 2.1. Upon multiplying (4) with its conjugate, and separating the diagonal summands from the off diagonal, for $x \in \mathbb{T}$ and $r>0$ we have that

$$
\int_{B_{x}(r)} f(y)^{2} d y=\pi r^{2}+\sum_{\lambda \neq \lambda^{\prime}} c_{\lambda} \overline{c_{\lambda^{\prime}}} \int_{B_{x}(r)} e\left(\left\langle\lambda-\lambda^{\prime}, y\right\rangle\right) d y .
$$

Therefore, transforming the variables $y=r \cdot z+x$ with $z \in B_{0}(1)$, we have

$$
\int_{B_{x}(r)} f(y)^{2} d y-\pi r^{2}=r^{2} \sum_{\lambda \neq \lambda^{\prime}} c_{\lambda} \overline{c_{\lambda^{\prime}}} e\left(\left\langle\lambda-\lambda^{\prime}, x\right\rangle\right) \int_{B(1)} e\left(\left\langle r\left(\lambda-\lambda^{\prime}\right), z\right\rangle\right) d z .
$$

where this time $B(1) \subseteq \mathbb{R}^{2}$ is the Euclidian centred unit ball. This yields the identity

$$
\int_{B_{x}(r)} f(y)^{2} d y-\pi r^{2}=r^{2} \sum_{\lambda \neq \lambda^{\prime}} c_{\lambda} \overline{c_{\lambda^{\prime}}} e\left(\left\langle\lambda-\lambda^{\prime}, x\right\rangle\right) \cdot \widehat{\chi}\left(r\left(\lambda-\lambda^{\prime}\right)\right),
$$


where $\chi$ is the characteristic of the unit disc. As $\chi$ is rotationally invariant so is its Fourier transform; a direct computation shows that its Fourier transform is given explicitly by

$$
\widehat{\chi}(\xi)=2 \pi \frac{J_{1}(\|\xi\|)}{\|\xi\|} .
$$

Substituting the latter into (33) yields the statement of Lemma 2.1.

Next we prove a strong version of Berry's conjecture for toral Laplace eigenfunctions.

Corollary 2.3. For almost all $n \in S$, if $f_{n}$ is as in (4), satisfying (6), and

$$
r \geq \frac{(\log n)^{1+\frac{\log 2}{3}+\epsilon}}{\sqrt{n}},
$$

then for all $x \in \mathbb{T}$ we have

$$
\int_{B_{x}(r)} f_{n}^{2} d y=\{\pi+o(1)\} r^{2} .
$$

Proof. If $n \in S$ then we can write $n=N m^{2}$, in which $m$ has only prime factors $\equiv 3$ (mod 4), and $N$ has no such prime factors, and then $r_{2}(n)=r_{2}(N)$. Note that if $N=2^{k} \ell$ where $\ell$ is odd, then $r_{2}(N)=4 \tau(\ell)$, where $\tau($.) is the divisor function. If $N$ is squarefree then $\tau(N)=4 \cdot 2^{\omega_{o}(N)}$ where $\omega_{o}(N)$ denotes the number of distinct odd prime factors of $N$. A famous result of Hardy and Ramanujan states that

$$
\omega(N)=\{1+o(1)\} \log \log N
$$

for almost all integers $N$. However our integers $N$ only have odd prime factors that are $\equiv 1(\bmod 4)$ so

$$
\omega(N)=\left\{\frac{1}{2}+o(1)\right\} \log \log N
$$

for almost all such integers $N \in S$. Since most integers have only a small part involving squares, one can then deduce that for almost all integers $n \in S$, one has

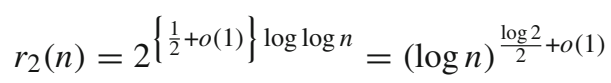

As discussed after its statement, Theorem 1.4 implies that for almost all $n \in S$, all $\lambda \neq \lambda^{\prime} \in \mathcal{E}_{n}$ satisfy

$$
\left\|\lambda-\lambda^{\prime}\right\| \gg \frac{\sqrt{n}}{(\log n)^{1+o(1)}} .
$$

The statement of Corollary 2.3 then follows upon substituting the above two results (34) and (35) into Corollary 2.2. 


\section{Limitations on Berry's Conjecture}

Next we prove the counterpoint to Corollary 2.2:

Proposition 3.1. For all $n \in S$ there exists an $f_{n}$ as in (4), satisfying (6), and a value of

$$
r \gg \frac{1}{\min _{\lambda \neq \lambda^{\prime}}\left\|\lambda-\lambda^{\prime}\right\|}
$$

for which

$$
\left|\int_{B_{0}(r)} f_{n}^{2} d y-\pi r^{2}\right| \gg r^{2} .
$$

In fact we get this lower bound for almost all $x \in \mathbb{T}$.

Proof. Select $\lambda \neq \lambda^{\prime} \in \mathcal{E}(n)$ for which $\left|\lambda-\lambda^{\prime}\right|$ is minimal. Let $c_{j}=0$ unless $j=\lambda, \lambda^{\prime}, \bar{\lambda}$ or $\overline{\lambda^{\prime}}$, in which case we have $c_{j}=1 / 2$ (with obvious modifications if $\lambda$ or $\lambda^{\prime} \in \mathbb{R}$ or $\left.\lambda^{\prime}=\bar{\lambda}\right)$. By Lemma 2.1, we then have

$$
\int_{B_{x}(r)} f_{n}^{2} d y-\pi r^{2}=\pi r^{2} \cos \left(2 \pi\left\langle x, \lambda-\lambda^{\prime}\right\rangle\right) \frac{J_{1}\left(r\left\|\lambda-\lambda^{\prime}\right\|\right)}{r\left\|\lambda-\lambda^{\prime}\right\|} .
$$

By (27) we deduce that there exists $r \asymp 1 /\left\|\lambda-\lambda^{\prime}\right\|$ for which

$$
\left|\int_{B_{x}(r)} f_{n}^{2} d y-\pi r^{2}\right| \asymp \pi r^{2}\left|\cos \left(2 \pi\left\langle x, \lambda-\lambda^{\prime}\right\rangle\right)\right| .
$$

The right-hand side will be big for most choices of $x$, but, in particular, taking $x=0$ we obtain

$$
\left|\int_{B_{0}(r)} f_{n}^{2} d y-\pi r^{2}\right| \asymp r^{2}
$$

Corollary 3.2. For almost all $n \in S$, there exists an $f_{n}$ as in (4), satisfying (6), and a value of

$$
r \geq \frac{(\log n)^{\frac{\log 2}{2}-\epsilon}}{\sqrt{n}}
$$

for which

$$
\int_{B_{0}(r)} f_{n}^{2} d y-\pi r^{2} \gg r^{2} .
$$

In fact we get this lower bound for almost all $x \in \mathbb{T}$. 
Proof. There are $r_{2}(n)$ elements of $\mathcal{E}(n)$ on a circle of perimeter $2 \pi \sqrt{n}$, and so

$$
\min _{\lambda \neq \lambda^{\prime}}\left\|\lambda-\lambda^{\prime}\right\|<2 \pi \cdot \frac{\sqrt{n}}{r_{2}(n)} .
$$

We substitute this bound into Proposition 3.1 to obtain the lower bound $r \gg r_{2}(n) / \sqrt{n}$ for all $n \in S$. The result now follows from (34).

Remark 3.3. We can infer from Corollaries 2.3 and 3.2 that our interpretation of Berry's conjecture is generically true for

$$
r>\frac{(\log n)^{A}}{\sqrt{n}},
$$

for any

$$
A>1+\frac{\log 2}{3}=1.23104906 \ldots
$$

and generically false for

$$
r>(\log n)^{B} / \sqrt{n}
$$

with

$$
B<\frac{\log 2}{2}=0.34657359 \ldots
$$

We would guess that there exists some critical exponent $C>0$ such that the conjecture is generically true for

$$
r>\frac{(\log n)^{A}}{\sqrt{n}}
$$

for every $A>C$, and is generically false for

$$
r \leq \frac{(\log n)^{B}}{\sqrt{n}}
$$

for every $B<C$. However we do not have a guess for the value of $C$.

It should be possible to improve Corollary 3.2 with the exponent

$$
\frac{\log 3}{2}=0.54930614 \ldots
$$

in place of $\frac{\log 2}{2}$, as follows: Almost all $n \in S$ can be written as $N m$ where $N$ is product of distinct primes $\equiv 1(\bmod 4)$, and $N$ has a particular structure: It consist of $\left(\frac{1}{2}-o(1)\right) \log \log n$ prime factors each of which lies in the interval

$$
\left[\exp \left((\log n)^{o(1)}\right), \exp \left((\log n)^{1-o(1)}\right)\right] .
$$

We split this interval into dyadic intervals, and run though the integers $N$ composed of such primes. If $p=a^{2}+b^{2}$ then the $a+i b$ should be more-or-less equidistributed in 
angle, so the set of elements of $\mathcal{E}(N)$, in which $N$ has exactly $k$ prime factors can be modelled by the random model

$$
\left\{\sqrt{N} e\left(\sum_{i=1}^{k} \delta_{i} \phi_{i}\right) ; \delta_{1}, \ldots, \delta_{k} \in\{-1,1\}\right\}
$$

where each $\phi_{i}$ is an iirv, uniformly distributed in $\mathbb{R} / \mathbb{Z}$.

Suppose that $\lambda, \lambda^{\prime}$ are the closest two elements of $\mathcal{E}_{N}$. If $|\alpha|^{2}=m$ then $\alpha \lambda, \alpha \lambda^{\prime} \in$ $\mathcal{E}(n)$, and

$$
\left|\alpha \lambda-\alpha \lambda^{\prime}\right| / \sqrt{n}=\left|\lambda-\lambda^{\prime}\right| / \sqrt{N} .
$$

Now this, according to the random model, should be roughly the expected value of the minimum of

$$
\left\{\left|\sum_{i=1}^{k} \eta_{i} \phi_{i}\right|: \eta_{1}, \ldots, \eta_{k} \in\{-1,0,1\}\right\}
$$

(where $2 \eta_{i}=\delta_{i}-\delta_{i}^{\prime}$ ). We can use Fourier analysis to ask for the expected number of such elements in an interval $[-\epsilon, \epsilon]$. As these are iirv's, all but the main term disappears, and so the answer as $2 \epsilon \cdot 3^{k}$. Therefore we should be able to take

$$
\epsilon \approx 3^{-k}=(\log n)^{-\frac{\log 3}{2}+o(1)}
$$

and so the claim. One would expect this to be unconditionally provable using the second moment method, though we leave this for other authors.

\section{Proof of Theorem 1.6 and Corollaries 1.7 and $\mathbf{1 . 1 0}$}

Lemma 4.1. Assume (6). The variance (19) of $X$ is given by

$$
\mathcal{V}(X)=8 \pi^{2} r^{4} \sum_{\lambda \neq \lambda^{\prime}}\left|c_{\lambda} c_{\lambda^{\prime}}\right|^{2} \frac{J_{1}\left(r\left\|\lambda-\lambda^{\prime}\right\|\right)^{2}}{r^{2}\left\|\lambda-\lambda^{\prime}\right\|^{2}} .
$$

Proof of Theorem 1.6 assuming Lemma 4.1. We invoke Lemma 4.1 and separate the near-diagonal terms

$$
0<\left|\lambda-\lambda^{\prime}\right|<\frac{1}{\xi r}
$$

from the rest to yield

$$
\begin{aligned}
\frac{\mathcal{V}(X)}{r^{4}} & \ll \sum_{0<\left|\lambda-\lambda^{\prime}\right|<\frac{1}{\xi r}}\left|c_{\lambda} c_{\lambda^{\prime}}\right|^{2} \frac{J_{1}\left(r^{2}\left\|\lambda-\lambda^{\prime}\right\|^{2}\right)}{r^{2}\left\|\lambda-\lambda^{\prime}\right\|^{2}} \\
& +\sum_{\left|\lambda-\lambda^{\prime}\right| \geq \frac{1}{\xi r}}\left|c_{\lambda} c_{\lambda^{\prime}}\right|^{2} \frac{J_{1}\left(r^{2}\left\|\lambda-\lambda^{\prime}\right\|^{2}\right)}{r^{2}\left\|\lambda-\lambda^{\prime}\right\|^{2}} .
\end{aligned}
$$


Upon using the bound $J_{1}(t) \ll t$ for the range $\left|\lambda-\lambda^{\prime}\right|<\frac{1}{\xi r}$, and the bound $J_{1}(t) \ll \frac{1}{\sqrt{t}}$ for $\left|\lambda-\lambda^{\prime}\right| \geq \frac{1}{\xi r}($ see (27)), we obtain the estimate

$$
\frac{\mathcal{V}(X)}{r^{4}} \ll \sum_{0<\left|\lambda-\lambda^{\prime}\right|<\frac{1}{\xi r}}\left|c_{\lambda} c_{\lambda^{\prime}}\right|^{2}+\sum_{\left|\lambda-\lambda^{\prime}\right| \geq \frac{1}{\xi r}}\left|c_{\lambda} c_{\lambda^{\prime}}\right|^{2} \frac{1}{r^{3}\left\|\lambda-\lambda^{\prime}\right\|^{3}} .
$$

For the latter summation in (36) we have

$$
\sum_{\left|\lambda-\lambda^{\prime}\right| \geq \frac{1}{\xi r}}\left|c_{\lambda} c_{\lambda^{\prime}}\right|^{2} \frac{1}{r^{3}\left\|\lambda-\lambda^{\prime}\right\|^{3}} \leq \xi^{3} \cdot \sum_{\lambda, \lambda^{\prime} \in \mathcal{E}_{n}}\left|c_{\lambda} c_{\lambda^{\prime}}\right|^{2}=\xi^{3}
$$

by (6). The result follows.

Proof of Lemma 4.1. We use the notation $X=X_{x}$ from (18). By Lemma 2.1 we have

$$
\begin{aligned}
\left(X_{x}-\mathbb{E}\left[X_{x}\right]\right)^{2}=4 \pi^{2} r^{4} \sum_{\lambda \neq \lambda^{\prime}, \lambda^{\prime \prime} \neq \lambda^{\prime \prime \prime}} c_{\lambda} \overline{c_{\lambda^{\prime}}} c_{\lambda^{\prime \prime}} \overline{c_{\lambda}^{\prime \prime \prime}} e\left(\left\langle x, \lambda-\lambda^{\prime}+\lambda^{\prime \prime}-\lambda^{\prime \prime \prime}\right\rangle\right) \times \\
\times \frac{J_{1}\left(r\left\|\lambda-\lambda^{\prime}\right\|\right) J_{1}\left(r\left\|\lambda^{\prime \prime}-\lambda^{\prime \prime \prime}\right\|\right)}{r^{2}\left\|\lambda-\lambda^{\prime}\right\| \cdot\left\|\lambda^{\prime \prime}-\lambda^{\prime \prime \prime}\right\|} .
\end{aligned}
$$

Integrating w.r.t. $x \in \mathbb{T}$ we are only left with the diagonal:

$$
\begin{aligned}
\mathcal{V}(X) & =\int_{\mathbb{T}}\left(X_{x}-\mathbb{E}\left[X_{x}\right]\right)^{2} d x \\
& =4 \pi^{2} r^{4} \sum_{\substack{\lambda \neq \lambda^{\prime}, \lambda^{\prime \prime} \neq \lambda^{\prime \prime \prime} \\
\lambda-\lambda^{\prime}+\lambda^{\prime \prime}-\lambda^{\prime \prime \prime}=0}} c_{\lambda} \overline{c_{\lambda^{\prime}}} c_{\lambda^{\prime \prime}} \overline{c_{\lambda}^{\prime \prime \prime}} \frac{J_{1}\left(r\left\|\lambda-\lambda^{\prime}\right\|\right) J_{1}\left(r\left\|\lambda^{\prime \prime}-\lambda^{\prime \prime \prime}\right\|\right)}{r^{2}\left\|\lambda-\lambda^{\prime}\right\| \cdot\left\|\lambda^{\prime \prime}-\lambda^{\prime \prime \prime}\right\|} .
\end{aligned}
$$

Now, as $\lambda \neq \lambda^{\prime}$ and $\lambda^{\prime \prime} \neq \lambda^{\prime \prime \prime}$ we have $\lambda-\lambda^{\prime}+\lambda^{\prime \prime}-\lambda^{\prime \prime \prime}=0$ if and only if either $\left(\lambda=-\lambda^{\prime \prime}\right.$ and $\left.\lambda^{\prime}=-\lambda^{\prime \prime \prime}\right)$ or $\left(\lambda=\lambda^{\prime \prime \prime}\right.$ and $\left.\lambda^{\prime}=\lambda^{\prime \prime}\right)$. Using this together with (5) we deduce the statement of Lemma 4.1.

\subsection{Proof of Corollaries 1.7 and 1.10.}

Proof of Corollary 1.7. An application of Theorem 1.6 with $\xi=\epsilon$ yields the bound

$$
\mathcal{V}(X) \ll\left(\sum_{0<\left|\lambda-\lambda^{\prime}\right|<\eta \sqrt{n}}\left|c_{\lambda}\right|^{2}\left|c_{\lambda^{\prime}}\right|^{2}+\epsilon^{3}\right) r^{4} .
$$

Now

$$
\sum_{0<\left|\lambda-\lambda^{\prime}\right|<\eta \sqrt{n}}\left|c_{\lambda}\right|^{2}\left|c_{\lambda^{\prime}}\right|^{2} \leq \sum_{\lambda \in \mathcal{E}_{n}}\left|c_{\lambda}\right|^{2} \sum_{\left|\lambda-\lambda^{\prime}\right|<\eta \sqrt{n}}\left|c_{\lambda^{\prime}}\right|^{2} \leq \epsilon \sum_{\lambda \in \mathcal{E}_{n}}\left|c_{\lambda}\right|^{2}=\epsilon,
$$

by (21) and (6). The statement of Corollary 1.7 then follows upon substitute the latter inequality into (38). 
Proof of Corollary 1.10. The assumption that $f$ is flat implies that (21) holds with $\eta=$ $n^{-\epsilon_{n}}$. A straightforward application of Corollary 1.7 with $\eta=n^{-\epsilon_{n}}$ and any fixed $\epsilon$ yields the first statement of this corollary.

For the second part we apply Theorem 1.6 with $\xi=r_{2}(n)^{-\epsilon}$ to yield the bound

$$
\mathcal{V}(X) \ll\left(\sum_{0<\left|\lambda-\lambda^{\prime}\right|<r_{2}(n)^{\epsilon} / r}\left|c_{\lambda} c_{\lambda^{\prime}}\right|^{2}+r_{2}(n)^{-3 \epsilon}\right) \cdot r^{4},
$$

and for $\epsilon$-ultraflat functions (26) we have

$$
\sum_{0<\left|\lambda-\lambda^{\prime}\right|<r_{2}(n)^{\epsilon} / r}\left|c_{\lambda} c_{\lambda^{\prime}}\right|^{2} \leq \frac{1}{r_{2}(n)^{2-2 \epsilon}} \#\left\{\lambda \neq \lambda^{\prime} \in \mathcal{E}_{n}:\left|\lambda-\lambda^{\prime}\right|<\frac{r_{2}(n)^{\epsilon}}{r}\right\} .
$$

For

$$
r \gg \frac{1}{n^{1 / 2-4 \epsilon}} \geq \frac{r_{2}(n)^{\epsilon}}{n^{1 / 2-3 \epsilon}}
$$

we may bound the latter as

$$
\begin{aligned}
& \#\left\{\lambda \neq \lambda^{\prime} \in \mathcal{E}_{n}:\left|\lambda-\lambda^{\prime}\right|<\frac{r_{2}(n)^{\epsilon}}{r}\right\} \\
& \leq \#\left\{\lambda \neq \lambda^{\prime} \in \mathcal{E}_{n}:\left|\lambda-\lambda^{\prime}\right|<n^{1 / 2-3 \epsilon}\right\} \ll r_{2}(n)^{2-3 \epsilon}
\end{aligned}
$$

by Theorem 1.8. The result finally follows upon substituting the latter estimate into (40), and then finally into (39).

\section{Close Lattice Points on a Given Circle}

Our goal is to prove Theorem 1.8. Our proof yields the more explicit upper bound,

$$
\#\left\{\alpha, \beta \in \mathcal{E}_{n}:|\alpha-\beta| \leq n^{1 / 2-\epsilon}\right\} \ll r_{2}(n)^{2-\epsilon}+\frac{1}{\epsilon} \cdot r_{2}(n)^{2-2 \epsilon} .
$$

In particular if $\epsilon=\frac{\log \log \left|\mathcal{E}_{n}\right|}{\log \left|\mathcal{E}_{n}\right|}$ the bound is

$$
\#\left\{\alpha, \beta \in \mathcal{E}_{n}:|\alpha-\beta| \leq n^{1 / 2-\epsilon}\right\} \ll \frac{r_{2}(n)^{2}}{\log r_{2}(n)} .
$$

We will also show, using the result of Cilleruelo and Cordoba [5], that we can replace the " $-\epsilon$ " in the exponent on the right-hand side of (22) by " $-\tau \epsilon$ " for any fixed $\tau<4$. 
5.1. The structure of the sets $\mathcal{E}_{n}$. If $\alpha \in \mathcal{E}_{n}$ then so are $u \alpha$ for each $u \in \mathcal{U}:=$ $\{1,-1, i,-i\}$, the set of units of $\mathbb{Z}[i]$. Note that there is therefore a unique $u \alpha=a+i b$ in first quadrant, so that $a>0$ and $b \geq 0$. We now describe the structure of the quotient set

$$
\mathcal{E}^{*}(n):=\mathcal{E}_{n} / \mathcal{U}
$$

The key observation is that these sets are multiplicative; that is,

$$
\mathcal{E}^{*}(m n)=\mathcal{E}^{*}(m) \cdot \mathcal{E}^{*}(n)
$$

if $(m, n)=1$ and all of the products are distinct, and so, in particular,

$$
r_{2}(m n) / 4=\left(r_{2}(m) / 4\right) \cdot\left(r_{2}(n) / 4\right) .
$$

Therefore to fully understand the sets $\mathcal{E}_{n}$ we need only focus on $\mathcal{E}^{*}\left(p^{k}\right)$. If $p \equiv 3$ $(\bmod 4)$ then $\mathcal{E}^{*}\left(p^{k}\right)=\emptyset$ if $k$ is odd, and $\mathcal{E}^{*}\left(p^{k}\right)=\left\{p^{k / 2}\right\}$ if $k$ is even. Also

$$
\mathcal{E}^{*}\left(2^{k}\right)=\left\{(1+i)^{k}\right\}
$$

for all $k \geq 1$. If $p \equiv 1(\bmod 4)$ then, as is well known, there are integers $a, b$, unique up to sign and swapping their order, for which $p=a^{2}+b^{2}$. Therefore if $P=a+i b$ then $\mathcal{E}^{*}(p)=\{P, \bar{P}\}$, and

$$
\mathcal{E}^{*}\left(p^{k}\right)=\left\{P^{k}, P^{k-1} \bar{P}, \ldots, P \bar{P}^{k-1}, \bar{P}^{k}\right\} .
$$

5.2. A first bound, using the structure. Throughout this section we will assume, without loss of generality, that

$$
n=\prod_{i=1}^{k} p_{i}^{n_{i}}
$$

where each $p_{j} \equiv 1(\bmod 4)$, at first in no particular order, then later for non-increasing $\left\{\frac{\log \left(n_{i}+1\right)}{n_{i} \log p_{i}}\right\}$ (see section 5.3).

Lemma 5.1. For every $n$ of the form (41), let $m$ be given by

$$
m=\prod_{i=1}^{\ell} p_{i}^{n_{i}}
$$

and $\theta \in \mathbb{R} / \mathbb{Z}$. The number of $\lambda \in \mathcal{E}_{n}$ satisfying

$$
\left|\lambda-\sqrt{n} e^{2 i \pi \theta}\right|<\sqrt{n / 2 m}
$$

$i s \leq 4 \prod_{i=\ell+1}^{k}\left(n_{i}+1\right)$. 
Proof. Suppose there are $>4 \prod_{i=\ell+1}^{k}\left(n_{i}+1\right)$ numbers $\lambda \in \mathcal{E}_{n}$ satisfying (43) for some $\theta \in \mathbb{R} / \mathbb{Z}$. We write each $\lambda \in \mathcal{E}_{n}$ as

$$
\lambda:=u \prod_{i=1}^{k} P_{i}^{e_{i}}{\overline{P_{i}}}^{n_{i}-e_{i}} .
$$

For at least two of the $\lambda$ with

$$
\left|\lambda-\sqrt{n} e^{2 i \pi \theta}\right|<\sqrt{n / 2 m},
$$

the $u$, and the $e_{i}$ are the same for all $i>\ell$, by the pigeonhole principle; we write the two numbers as $\lambda=\gamma \beta$ and $\lambda^{\prime}=\gamma \beta^{\prime}$ where

$$
\gamma:=u \prod_{i=\ell+1}^{k} P_{i}^{e_{i}}{\overline{P_{i}}}^{n_{i}-e_{i}} .
$$

Now $\beta-\beta^{\prime} \in \mathbb{Z}[i]$ with $|\beta|=\left|\beta^{\prime}\right|$ so that $\left|\beta-\beta^{\prime}\right| \geq \sqrt{2}$. Therefore

$$
\begin{aligned}
2 \sqrt{n / 2 m} & >\left|\lambda-\sqrt{n} e^{2 i \pi \theta}\right|+\left|\lambda^{\prime}-\sqrt{n} e^{2 i \pi \theta}\right| \geq\left|\lambda-\lambda^{\prime}\right| \\
& \geq \sqrt{2}|\gamma|=\sqrt{2} \prod_{i=\ell+1}^{k} p_{i}^{n_{i} / 2}=\sqrt{2 n / m},
\end{aligned}
$$

a contradiction.

We can revisit Lemma 5.1 putting a better lower bound on $\left|\beta-\beta^{\prime}\right|$ by using CillerueloCordoba [5]:

Lemma 5.2. Fix $\epsilon>0$, and let $n$ be of the form (41) and $m$ of the form (42). Then for every $\theta \in \mathbb{R} / \mathbb{Z}$ the number of $\lambda \in \mathcal{E}_{n}$ satisfying

$$
\left|\lambda-\sqrt{n} e^{2 i \pi \theta}\right|<\sqrt{n} / m^{1 / 4-\epsilon}
$$

is $\ll(1 / \epsilon) \tau(n / m)$.

Proof. Cilleruelo and Cordoba [5] proved that an arc on a circle of radius $R$, which contains more than $2 r$ lattice points, has length $>2^{1 / 2} R^{r /(2 r+1)}$. Therefore if our arc in the proof of Lemma 5.1, contains $>8 r \prod_{i=\ell+1}^{k}\left(n_{i}+1\right)$ numbers $\lambda \in \mathcal{E}_{n}$, then we have more than $2 r$ with the same $\gamma$, and so more than $2 r$ lattice points $\beta$ lie on a circle of radius $m^{1 / 2}$. Thus the $\beta$-arc has width $>2^{1 / 2} m^{r / 2(2 r+1)}$, and after multiplying by $\gamma$ this transforms to an arc of width

$$
>2^{1 / 2} \sqrt{n / m} \cdot m^{r / 2(2 r+1)}=\frac{(2 n)^{1 / 2}}{m^{(r+1) / 2(2 r+1)}}
$$

on the radius $-\sqrt{n}$ circle.

We therefore deduce that on the radius- $\sqrt{n}$ circle any arc of width $\leq \frac{(2 n)^{1 / 2}}{m^{(r+1) / 2(2 r+1)}}$ contains no more than $8 r \prod_{i=\ell+1}^{k}\left(n_{i}+1\right)$ numbers $\lambda \in \mathcal{E}_{n}$. Hence, upon losing a factor $\frac{1}{2}$ via a triangle inequality similar to (44), the number of $\lambda \in \mathcal{E}_{n}$ with

$$
\left|\lambda-\sqrt{n} e^{2 i \pi \theta}\right|<\frac{1}{2} \frac{(2 n)^{1 / 2}}{m^{(r+1) / 2(2 r+1)}}=\frac{(n / 2)^{1 / 2}}{m^{(r+1) / 2(2 r+1)}}
$$


is also

$$
\leq 8 r \prod_{i=\ell+1}^{k}\left(n_{i}+1\right),
$$

which is $\leq n^{1 / 2} / m^{1 / 4-\epsilon}$ if $r \gg 1 / \epsilon$.

5.3. Balancing a prime power and its power. For the rest of this section we will organize the $p_{i}$ so that the $\frac{\log \left(n_{i}+1\right)}{n_{i} \log p_{i}}$ are non-increasing. This will allow us to generalize the above argument to the case in which $m$ and $n / m$ are not necessarily coprime. We have the following corollary of Lemma 5.1.

Corollary 5.3. Let $n$ be of the form (41) and $m$ of the form (42) so that $(m, n / m)=1$, with $l$ chosen to be the largest integer for which

$$
m \leq n^{2 \epsilon} / 2 \text {. }
$$

If $m \geq n^{\epsilon}$ or $n_{\ell+1} \ll 1 / \epsilon$ then

$$
\#\left\{(u, v) \in \mathcal{E}_{n}:|u-v| \leq n^{1 / 2-\epsilon}\right\} \ll_{\epsilon}\left|\mathcal{E}_{n}\right|^{2-\epsilon}+\frac{1}{\epsilon} \cdot\left|\mathcal{E}_{n}\right|^{2-2 \epsilon} .
$$

Proof. Putting

$$
m^{*}=m p_{\ell+1}^{n_{\ell+1}}
$$

we have $m^{*}>n^{2 \epsilon} / 2$ by the definition of $l$, and hence $m$. Define $\delta$ so that $n / m=n^{\delta}$. We claim that $\tau(n / m) \leq \tau(n)^{\delta}$. To see this define the real numbers $e_{i}$ to satisfy the equation $n_{i}+1=\left(p_{i}^{n_{i}}\right)^{e_{i}}$; the $e_{i}$ are ordered so that $e_{1} \geq e_{2} \geq \ldots$. Then $\tau(n / m)=$ $\prod_{i=\ell+1}^{k}\left(p_{i}^{n_{i}}\right)^{e_{i}}=(n / m)^{E}$ say, and $\tau(m)=m^{F}$, where $E \leq e_{\ell+1} \leq e_{\ell} \leq F$. Now $(n / m, m)=1$ and so $\tau(n)=\tau(n / m) \tau(m)=(n / m)^{E} m^{F}=n^{G}$ where $E \leq G \leq F$. Therefore $\tau(n / M)=(n / m)^{E}=n^{\delta E} \leq n^{\delta G}=\tau(n)^{\delta}$, and the claim follows

Now $n / m^{*} \leq 2 n^{1-2 \epsilon}$ and so $\tau\left(n / m^{*}\right) \leq 2 \tau(n)^{1-2 \epsilon}$, by the argument in the first paragraph. This implies

$$
\tau(n / m)=\tau\left(n / m^{*}\right)\left(n_{\ell+1}+1\right) \ll \tau(n)^{1-2 \epsilon} / \epsilon,
$$

assuming that $n_{\ell+1} \ll 1 / \epsilon$.

Otherwise $m \geq n^{\epsilon}$ in which case $n / m \leq n^{1-\epsilon}$, and so $\tau(n / m) \leq \tau(n)^{1-\epsilon}$, by the first paragraph. Therefore, by (45) and Lemma 5.1,

$$
\begin{aligned}
& \#\left\{(u, v) \in \mathcal{E}_{n}:|u-v| \leq n^{1 / 2-\epsilon}\right\}=\sum_{u \in \mathcal{E}_{n}} \#\left\{v \in \mathcal{E}_{n}:|u-v| \leq n^{1 / 2-\epsilon}\right\} \\
& \leq r_{2}(n) \max _{\theta \in \mathbb{R} / \mathbb{Z}} \#\left\{v \in \mathcal{E}_{n}:\left|v-\sqrt{n} e^{2 i \pi \theta}\right| \leq \sqrt{n / 2 m}\right\} \leq r_{2}(n) \cdot 4 \tau(n / m),
\end{aligned}
$$

and the result follows from the bounds on $\tau(n / m)$ given above. 
Proof of Theorem 1.8. Corollary 5.3 yields the result at once, unless $m<n^{\epsilon}$ and $n_{\ell+1} \geq$ $10 / \epsilon$. In this case $p_{\ell+1}^{n_{\ell+1}}>n^{\epsilon} / 2$, else $n^{2 \epsilon} / 2<m^{*}=m p_{\ell+1}^{n_{\ell+1}} \leq m n^{\epsilon} / 2$, so that $m \geq n^{\epsilon}$. For ease of notation, we write $q=p_{\ell+1}$ corresponding to the Gaussian prime $Q=P_{\ell+1}$, and $N=n_{\ell+1}$, so that $m^{*}=m q^{N}$ :

We let $d$ be the largest integer for which

$$
m^{\dagger}:=m q^{d} \leq n^{2 \epsilon} / 2 .
$$

We now show that $\epsilon N / 2<d<N$ : first we recall that by the definition of $m^{*}$ we have that $m^{*}>n^{2 \epsilon} / 2$, hence, by the definition of $N$, we have $d<N$. Now $N \geq 10 / \epsilon$, and $q^{N} \leq n$, so $q<n^{\epsilon / 10}$. By definition $q^{d+1}>n^{2 \epsilon} / 2 m>n^{\epsilon} / 2>q^{10} / 2$, and so $d \geq 9$. Moreover

$$
\left(q^{d+1}\right)^{1 / \epsilon+1}>\left(n^{\epsilon} / 2\right)^{1 / \epsilon+1} \geq n \geq q^{N},
$$

and so $d \geq \epsilon N / 2$

For a given integer $d$, let $w$ be the smallest integer with $w d \geq N$. If we have $w+1$ integers amongst $0, \ldots, N$ then two of them differ by at most $d$. We have $w \leq 2 / \epsilon+1$ since $d>\epsilon N / 2$.

We now prove that there are $\leq 4(w+1) \tau\left(n / m^{*}\right)$ numbers $\alpha \in \mathcal{E}_{n}$ with

$$
\left|\alpha-\sqrt{n} e^{2 i \pi \theta}\right|<\sqrt{n / 2 m^{\dagger}}
$$

for every $\theta \in \mathbb{R} / \mathbb{Z}$ : For if not then we have $\alpha, \alpha^{\prime}$ with $e_{i}=e_{i}^{\prime}$ for all $i>\ell+1$, and $u=u^{\prime}$, but the exponents of $Q$ and $\bar{Q}$ are $Q^{e} \bar{Q}^{n-e}$ and $Q^{e+\Delta} \bar{Q}^{n-e-\Delta}$, for some $\Delta, 0 \leq \Delta \leq d$. The contribution to $\gamma$ is therefore $Q^{e} \bar{Q}^{n-e-\Delta}$ which has norm $q^{\frac{n-\Delta}{2}} \geq q^{\frac{n-d}{2}}$. Therefore $|\gamma| \geq \sqrt{n / m^{\dagger}}$. We recover the same contradiction as in Lemma 5.1.

Proceeding as in the proof of Corollary 5.3, and recalling from these that $\tau\left(n / m^{*}\right) \leq$ $2 \tau(n)^{1-2 \epsilon} \leq 2 r_{2}(n)^{1-2 \epsilon}$, we then deduce that

$$
\#\left\{(u, v) \in \mathcal{E}_{n}:|u-v| \leq n^{1 / 2-\epsilon}\right\} \leq 8(w+1) \tau(n)^{2-2 \epsilon} \ll \frac{1}{\epsilon} \cdot\left|\mathcal{E}_{n}\right|^{2-2 \epsilon} .
$$

We have proved both Theorem 1.8 and the claim (23).

Remark 5.4. We can improve Theorem 1.8 unconditionally to

$$
\#\left\{(u, v) \in \mathcal{E}_{n}:|u-v| \leq n^{1 / 2-\epsilon}\right\} \ll_{\epsilon}\left|\mathcal{E}_{n}\right|^{2-\tau \epsilon},
$$

for any fixed $\tau<4$, by choosing $\ell$ so that $m \leq n^{\tau \epsilon}$, and using Lemma 5.2 in place of Lemma 5.1 in the proof above.

5.4. On conjectured bounds for lattice points in short arcs. In Conjecture 15 of [6] it is conjectured that for any fixed $\epsilon>0$, there are $\ll_{\epsilon} 1$ lattice points on an arc of length $R^{1-\epsilon}$ of a circle of radius $R$, in which case the upper bound in Theorem 1.8 would be $\ll\left|\mathcal{E}_{n}\right|$, for any fixed $\epsilon>0$.

In the special case that $n=p^{g}$ is a prime power, we can use ideas of Diophantine approximation to lower bound $\left|\operatorname{Im}\left((a+i b)^{g}\right)\right|$ where $a^{2}+b^{2}=p$ : Let

$$
f(t):=\frac{1}{2 i}\left((t+i)^{g}-(t-i)^{g}\right)
$$


so that

$$
\operatorname{Im}\left((a+i b)^{g}\right)=F(a, b)
$$

where $F(x, y):=y^{g} f(x / y)$ is a homogenous polynomial of degree $g$. Now

$$
2 i f^{\prime}(t)=g\left((t+i)^{g-1}-(t-i)^{g-1}\right)
$$

and so $\left(f, f^{\prime}\right)=1$. This implies that $f$ has no repeated roots, and that $F$ has no repeated factors. that

Roth's Theorem gives that $|f(a / b)| \gg_{g, \eta} 1 /|b|^{2+2 \eta}$, for each fixed $g$, which implies

$$
\operatorname{Im}\left(P^{g}\right) \gg_{g, \epsilon} p^{g / 2} /|b|^{2+2 \epsilon} \geq p^{g / 2-1-\epsilon} .
$$

We can obtain a uniform version of this result by using the $a b c$-conjecture in the field $\mathbb{Z}[i]$ (see [8]): Suppose that $a+b=c$ with $a, b, c$ coprime elements of $\mathbb{Z}[i]$. Then

$$
\prod_{Q \mid a b c}|Q| \gg_{\epsilon} \max \{|a|,|b|,|c|\}^{1-\epsilon},
$$

where the product runs over the distinct primes $Q$ in $\mathbb{Z}[i]$. We have the equation

$$
(a+b i)^{g}-(a-b i)^{g}=2 i \operatorname{Im}\left(P^{g}\right),
$$

and the terms are coprime if $p>2$. The $a b c$-conjecture then implies

$$
\begin{aligned}
\operatorname{Im}\left(P^{g}\right) p & =\operatorname{Im}\left(P^{g}\right)(a+b i)(a-b i) \geq \prod_{Q \mid(a+b i)^{g}(a-b i)^{g} \cdot \operatorname{Im}\left(P^{g}\right)}|Q| \\
& \gg_{\epsilon}\left(|a+i b|^{g}\right)^{1-\epsilon}=\left(p^{g / 2}\right)^{1-\epsilon},
\end{aligned}
$$

and therefore we recover (47), in which the implicit constant is independent of $g$.

\section{Pairs of Close-By Lattice Points, Over All Radii $\leq \sqrt{N}$}

6.1. Reformulation, and automorphisms of pairs of close lattice points. If $a, b \in \mathcal{E}_{n}$ and $|a-b| \leq M$ then let $\alpha=\operatorname{gcd}(a, b)$ (determined up to a unit, cf. (52) below) in $\mathbb{Z}[i]$, and $\beta=a / \alpha$. Then $b=u \alpha \bar{\beta}$ where $(\beta, \bar{\beta})=1$ so that $\beta$ is not divisible by any integer $>1$ and $u \in \mathcal{U}$. Therefore the elements of $S(n, M)$, defined as the set

$S(n, M):=\left\{(\alpha, \beta, u) \in \mathbb{Z}[i]^{2} \times \mathcal{U}:|\alpha| \cdot|\beta|=\sqrt{n},|\alpha| \cdot|\beta-u \bar{\beta}| \leq M,(\beta, \bar{\beta})=1\right\}$, are in 1-to-1 correspondence with the pairs

$$
\left\{(a, b) \in \mathcal{E}_{n}:|a-b| \leq M\right\} .
$$

Our goal is to estimate the number of exceptions to $B R(\delta)$, for given $\delta>0$. More generally, given $N$ and $M \leq 2 \sqrt{N}$ define the set of close pairs

$$
\mathcal{G}(N ; M)=\left\{\left(\lambda, \lambda^{\prime}\right):\|\lambda\|^{2}=\left\|\lambda^{\prime}\right\|^{2} \leq N, 0<\left\|\lambda-\lambda^{\prime}\right\|<M\right\} .
$$


Define the function $I(c):[0,1] \rightarrow \mathbb{R}$ by

$$
I(c):=\frac{4}{\pi} \int_{0}^{1}\left(1-c t^{2}\right)^{1 / 2} d t
$$

Note that $I(c)$ is decreasing from $I(0)=\frac{4}{\pi}$ to $I(1)=1$, as $c$ goes from 0 to 1 . Moreover

$$
I(c)=\frac{4}{\pi}+O(c) .
$$

Theorem 6.1. Let $N \rightarrow \infty$ be a large parameter, $M=M(N)$, and $\mathcal{G}(N ; M)$ defined in (48). We have

$$
\mathcal{G}(N ; M)=4 \cdot I\left(\frac{M^{2}}{4 N}\right) \cdot \sqrt{N} M \log M \cdot\left(1+O\left(\frac{1}{\log M}\right)\right),
$$

which is an asymptotic as long as $M \rightarrow \infty$.

The proof of Theorem 6.1 will be given in section 6.2 .

Proof of Theorem 1.3 assuming Theorem 6.1. Let $J=[\sqrt{\log N}]$ and select $\epsilon>0$ so that

$$
(1-\epsilon)^{J}=1 / 2 \text {. }
$$

We write $N^{\prime}=(1-\epsilon) N$ with $L=C N^{1 / 2-\delta}$ and $L^{\prime}=C\left(N^{\prime}\right)^{1 / 2-\delta}$, and $C>0$ is the constant in definition (13) of $B^{*}(N ; \delta)$. By the definitions (13) and (48) of $B^{*}(\cdot ; \cdot)$ and $\mathcal{G}(\cdot ; \cdot)$ respectively, we have

$$
\mathcal{G}\left(N ; L^{\prime}\right) \leq \mathcal{G}(N ; L)=B^{*}(N ; \delta)
$$

and

$$
\mathcal{G}\left(N^{\prime} ; L^{\prime}\right)=B^{*}\left(N^{\prime} ; \delta\right) \leq \mathcal{G}\left(N^{\prime} ; L\right)
$$

we have that

$$
\mathcal{G}\left(N ; L^{\prime}\right)-\mathcal{G}\left(N^{\prime} ; L^{\prime}\right) \leq B^{*}(N ; \delta)-B^{*}\left(N^{\prime} ; \delta\right) \leq \mathcal{G}(N ; L)-\mathcal{G}\left(N^{\prime} ; L\right) .
$$

Substituting in the estimate from Theorem 6.1 we obtain

$$
B^{*}(N ; \delta)-B^{*}((1-\epsilon) N ; \delta)=\frac{4 C}{\pi}(1-2 \delta) N^{1-\delta} \log N \cdot \epsilon(1+O(\epsilon)) .
$$

Replacing $N$ by $(1-\epsilon)^{j} N$ for $j=0,1,2, \ldots, J-1$ and summing, we obtain

$$
B^{*}(N ; \delta)-B^{*}(N / 2 ; \delta)=\frac{4 C}{\pi}(1-2 \delta) \cdot \frac{N^{1-\delta}-(N / 2)^{1-\delta}}{(1-\delta) \epsilon(1+O(\epsilon))} \log N \cdot \epsilon(1+O(\epsilon))
$$

Finally replacing $N$ by $N / 2^{j}$ for $j=0,1,2, \ldots$ and summing, we obtain the claimed result. 


\subsection{The number of close-by pairs.}

Proof of Theorem 6.1. We will use the "if and only if" criterion above, so we wish to count

$$
\begin{aligned}
\mathcal{G}(N, M) & =\sum_{n \leq N}|S(n, M)| \\
& =\frac{1}{4} \sum_{u \in\{1, i,-1,-i\}} \#\left\{\alpha, \beta \in \mathbb{Z}[i]:|\beta| \leq \frac{\sqrt{N}}{|\alpha|},|\beta-u \bar{\beta}| \leq \frac{M}{|\alpha|}, \quad(\beta, \bar{\beta})=1\right\},
\end{aligned}
$$

since $\alpha$ is determined up to a unit. If $u=1$ then $|\beta-u \bar{\beta}|=2|\operatorname{Im}(\beta)|$, so if we write $\beta=x+i y$, the condition $(\beta, \bar{\beta})=1$ is equivalent to $(x, y)=1$ and $x+y$ is odd (note that $(1+i)^{2}=2 i$ so that $1+i$ is a prime factor of 2 in $\mathbb{Z}[i]$, and so if $x+y$ is even then $1+i=i(1-i)$ would divide both $\beta$ and $\bar{\beta})$, the conditions on the r.h.s. of (52) are

$$
|y| \leq M / 2|\alpha| \text { and } x^{2} \leq N /|\alpha|^{2}-y^{2} \text {, with }(x, y)=1 \text { and } x+y \text { odd }
$$

To count this we fix $y$ and vary over $x$. Now $x$ runs through an interval of length $X$, say. Moreover

$$
x \equiv y+1 \quad(\bmod 2)
$$

and $(x, y)=1$. Hence, by the inclusion exclusion principle, given $y$, the number of $x$ satisfying (53) is

$$
\frac{\varphi(2 y)}{2 y} \cdot X+O(\tau(y))
$$

where $\tau(y)$ denotes the number of squarefree divisors of $y$. Therefore, in total, the summand on the r.h.s. of (52) corresponding to $u=1$ equals

$$
\begin{aligned}
& \#\left\{\alpha, \beta \in \mathbb{Z}[i]:|\beta| \leq \frac{\sqrt{N}}{|\alpha|},|\beta-\bar{\beta}| \leq \frac{M}{|\alpha|}, \quad(\beta, \bar{\beta})=1\right\} \\
& =4 \sum_{|\alpha| \leq M / 2} \sum_{1 \leq y \leq M / 2|\alpha|} \frac{\varphi(2 y)}{2 y} \cdot\left(\frac{N}{|\alpha|^{2}}-y^{2}\right)^{1 / 2}+O\left(\sum_{|\alpha| \leq M / 2} \sum_{|y| \leq M / 2|\alpha|} \tau(y)\right) .
\end{aligned}
$$

The inner summation of the error terms on the r.h.s. of (54) is

$$
\sum_{y:|y| \leq M / 2|\alpha|} \tau(y) \ll \frac{M}{2|\alpha|} \cdot \log (M / 2|\alpha|) .
$$

Now, the number of such $\alpha$ with $\frac{M}{2^{k+1}}<|\alpha| \leq \frac{M}{2^{k}}$ is $\ll \frac{M^{2}}{2^{2 k}}$, so our bound for the total error term in (54) is

$$
\sum_{M / 2^{k+1}<|\alpha| \leq M / 2^{k}} \sum_{|y| \leq M / 2|\alpha|} \tau(y) \ll \sum_{k \geq 1} \frac{M^{2}}{2^{2 k}} \cdot 2^{k} k \ll M^{2} .
$$


Substituting the latter into (54) it reads (this is the summand in (52) corresponding to $u=1$ )

$$
\begin{aligned}
& \#\left\{\alpha, \beta \in \mathbb{Z}[i]:|\beta| \leq \frac{\sqrt{N}}{|\alpha|},|\beta-\bar{\beta}| \leq \frac{M}{|\alpha|}, \quad(\beta, \bar{\beta})=1\right\} \\
& =4 \sum_{y: 1 \leq y \leq M / 2} \frac{\varphi(2 y)}{2 y} \sum_{\substack{\alpha \in \mathbb{Z}[i] \\
|\alpha| \leq M / 2 y}}\left(\frac{N}{|\alpha|^{2}}-y^{2}\right)^{1 / 2}+O\left(M^{2}\right) .
\end{aligned}
$$

Now define $R(t):=\sum_{\alpha \in \mathbb{Z}[i],|\alpha| \leq T} 1=\pi T^{2}+O(T)$, and use summation by parts to evaluate the inner sum on the r.h.s. of (55). We have

$$
\begin{aligned}
& \sum_{\substack{\alpha \in \mathbb{Z}[i] \\
|\alpha| \leq M / 2 y}}\left(\frac{N}{|\alpha|^{2}}-y^{2}\right)^{1 / 2}=\int_{1}^{M / 2 y}\left(\frac{N}{t^{2}}-y^{2}\right)^{1 / 2} d R(t) \\
& =\int_{1}^{M / 2 y}\left(\frac{N}{t^{2}}-y^{2}\right)^{1 / 2} d\left(\pi t^{2}+O(t)\right) \\
& =2 \pi N^{1 / 2} \int_{1}^{M / 2 y}\left(1-\frac{y^{2} t^{2}}{N}\right)^{1 / 2} d t+O\left(N^{1 / 2} \log (M / 2 y)\right),
\end{aligned}
$$

where the above formal treatment in the last equality in (56) hides applying summation by parts followed by integration by parts in "opposite direction", noting that the boundary terms cancel each other upon the sequential applications of the summation by parts, and the relevant summands are of the same sign (so that we can differentiate the error term). To evaluate the integral on the r.h.s. of (56) we transform the variables $M v=2 y t$, so that

$$
\begin{aligned}
& \int_{1}^{M / 2 y}\left(1-\frac{y^{2} t^{2}}{N}\right)^{1 / 2} d t=\frac{M}{2 y} \int_{2 y / M}^{1}\left(1-\frac{M^{2}}{4 N} v^{2}\right)^{1 / 2} \\
& d v=\frac{M}{2 y}\left(I\left(\frac{M^{2}}{4 N}\right)+O\left(\frac{y}{M}\right)\right),
\end{aligned}
$$

upon extending the range of the integral and recalling the definition (49) of $I(c)$. Substituting the latter into (56) yields

$$
\sum_{\substack{\alpha \in \mathbb{Z}[i] \\|\alpha| \leq M / 2 y}}\left(\frac{N}{|\alpha|^{2}}-y^{2}\right)^{1 / 2}=\frac{\pi^{2} N^{1 / 2} M}{4 y} I\left(\frac{M^{2}}{4 N}\right)+O\left(N^{1 / 2}\left(\log \left(\frac{M}{2 y}\right)+1\right)\right) .
$$

We then find that the sum of the error terms on the r.h.s. of (57) along the range of summation of (55) is bounded by 


$$
\ll N^{1 / 2} \sum_{y: 1 \leq y \leq M / 2} \log \left(\frac{M}{2 y}\right)+N^{1 / 2} M \ll N^{1 / 2} M
$$

by comparing the summation in (58) to the corresponding integral.

Now we substitute the estimate (57) into (55), and use the bound (58) for the relevant summation of the error terms to obtain

$$
\begin{aligned}
& \#\left\{\alpha, \beta \in \mathbb{Z}[i]:|\beta| \leq \frac{\sqrt{N}}{|\alpha|},|\beta-\bar{\beta}| \leq \frac{M}{|\alpha|}, \quad(\beta, \bar{\beta})=1\right\} \\
& =\pi^{2} N^{1 / 2} M \cdot I\left(\frac{M^{2}}{4 N}\right) \cdot \sum_{y: 1 \leq y \leq M / 2} \frac{\varphi(2 y)}{2 y^{2}}+O\left(N^{1 / 2} M\right),
\end{aligned}
$$

where the error term $O\left(N^{1 / 2} M\right)$ also encapsulates $O\left(M^{2}\right)$ from (55), as $M \leq 2 \sqrt{N}$.

For the main term on the r.h.s. of (59) we need to determine

$$
\begin{aligned}
\sum_{y: 1 \leq y \leq M / 2} \frac{\varphi(2 y)}{2 y^{2}} & =\frac{1}{2} \sum_{\begin{array}{c}
y: 1 \leq y \leq M / 2 \\
y
\end{array}} \frac{1}{y} \sum_{\substack{d \mid y \\
d \text { odd }}} \frac{\mu(d)}{d}=\frac{1}{2} \sum_{\substack{d \leq M / 2 \\
d \text { odd }}} \frac{\mu(d)}{d} \sum_{y \leq M / 2, d \mid y} \frac{1}{y} \\
& =\frac{1}{2} \sum_{\substack{d \leq M / 2 \\
d \leq \text { odd }}} \frac{\mu(d)}{d^{2}} \sum_{m \leq M / 2 d} \frac{1}{m} \\
& =\frac{1}{2} \sum_{\substack{d \leq M / 2 \\
d}} \frac{\mu(d)}{d^{2}}(\log (M / 2 d)+\gamma+O(d / M)) \\
& =\frac{1}{2} \sum_{\substack{d \leq M / 2 \\
d}} \frac{\mu(d)}{d^{2}} \log (M / 2 d)+\frac{\gamma}{2} \sum_{\substack{d \leq M / 2 \\
d}} \frac{\mu(d)}{d^{2}}+O\left(\frac{\log M}{M}\right),
\end{aligned}
$$

writing $y=d m$. Now

$$
\sum_{\substack{d \leq M / 2 \\ d \text { odd }}} \frac{\mu(d)}{d^{2}}=\sum_{d \text { odd }, \geq 1} \frac{\mu(d)}{d^{2}}+O\left(\sum_{d>M / 2} \frac{1}{d^{2}}\right)=\frac{8}{\pi^{2}}+O(1 / M) .
$$

Also

$$
\sum_{d \text { odd }} \frac{\mu(d) \log d}{d^{2}}=\sum_{d \text { odd }} \frac{\mu(d)}{d^{2}} \sum_{p \mid d} \log p=\sum_{p \text { odd }} \log p \sum_{\substack{d \text { odd } \\ p \mid d}} \frac{\mu(d)}{d^{2}}=-\frac{8}{\pi^{2}} \sum_{p \text { odd }} \frac{\log p}{p^{2}-1} .
$$

Combining the estimates (61) and (62) and inserting them into (60) gives

$$
\begin{aligned}
\sum_{y: 1 \leq y \leq M / 2} \frac{\varphi(2 y)}{2 y^{2}} & =\frac{4}{\pi^{2}}\left(\log M / 2+\gamma+\sum_{p \text { odd }} \frac{\log p}{p^{2}-1}\right)+O\left(\frac{\log M}{M}\right) \\
& =\frac{4}{\pi^{2}} \log M \cdot\left(1+O\left(\frac{1}{\log M}\right)\right),
\end{aligned}
$$


so that (59) is

$$
\begin{aligned}
& \#\left\{\alpha, \beta \in \mathbb{Z}[i]:|\beta| \leq \frac{\sqrt{N}}{|\alpha|},|\beta-\bar{\beta}| \leq \frac{M}{|\alpha|}\right\} \\
& =4 N^{1 / 2} M \log M \cdot I\left(\frac{M^{2}}{4 N}\right) \cdot\left(1+O\left(\frac{1}{\log M}\right)\right),
\end{aligned}
$$

where the error term in the latter estimate also encapsulates the one in (59). The estimate (64) means that the term in the sum on the r.h.s. of (52) corresponding to $u=1$ contributes $\frac{1}{4}$ of what is claimed in the statement (51) of Theorem 6.1.

We claim that the contribution of each of the other three terms in (52) is also given by the r.h.s. of (64). While the proofs are very similar we highlight the differences for the convenience of the reader. The $u=-1$ term yields the conditions

$$
|x| \leq M / 2|\alpha| \text { and } y^{2} \leq N /|\alpha|^{2}-x^{2} \text {, with }(x, y)=1 \text { and } x+y \text { odd }
$$

that is, the roles of $x$ and $y$ are reversed as compared to (53); one then gets the same estimate. If $u=i$ then $\beta-u \bar{\beta}=(1-i)(x-y)$, so let $y=x+\Delta$ so that $x^{2}+y^{2} \leq T^{2}$ becomes $(2 x+\Delta)^{2} \leq 2 T^{2}-\Delta^{2}$. Therefore we have the conditions, for $X=\left(2 N /|\bar{\alpha}|^{2}-\right.$ $\left.\Delta^{2}\right)^{1 / 2}$

$$
\begin{array}{r}
|\Delta| \leq M / \sqrt{2}|\alpha| \text { and } \frac{-X-\Delta}{2} \leq x \leq \frac{X-\Delta}{2} \\
\text { with }(x, \Delta)=1 \text { and } \Delta \text { odd }
\end{array}
$$

We now have a slightly different calculation from before; we will note the differences: Again $x$ runs through an interval of length $X$, and so the number of such $x$ is

$$
\frac{\varphi(\Delta)}{\Delta} \cdot X+O(\tau(\Delta)) \text {. }
$$

Running through the calculation we get a main term of

$$
\sum_{\substack{\Delta \leq M / \sqrt{2} \\ \Delta \text { odd }}} \frac{\varphi(\Delta)}{\Delta^{2}} \cdot \frac{\pi N^{1 / 2} M}{y}
$$

with the same error terms. An analogous calculation reveals that

$$
\sum_{\substack{\Delta \leq M / \sqrt{2} \\ \Delta \text { odd }}} \frac{\varphi(\Delta)}{\Delta^{2}}=\frac{4}{\pi^{2}}\left(\log \sqrt{2} M+\gamma+\sum_{p \text { odd }} \frac{\log p}{p^{2}-1}\right)+O\left(\frac{\log M}{M}\right) .
$$

A similar calculation ensues for $u=-i$. Therefore, as mentioned above and similar to (64), each summand of (52) contribute a quarter of the total claimed (51), and the result follows. 
6.3. Automorphisms of pairs of close lattice points. We observe that if $(\alpha, \beta, u) \in$ $S(n, M)$ corresponds to $(a, b) \in \mathcal{E}_{n}$ then, taking conjugates,

$$
(\bar{\alpha}, \bar{\beta}, \bar{u}) \in S(n, M)
$$

corresponds to $(\bar{a}, \bar{b}) \in \mathcal{E}_{n}$. More interestingly, given $(\alpha, \beta, u) \in S(n, M)$ we see that

$$
A(\alpha, \beta, u):=\left\{\left(\alpha^{\prime}, \beta w, u w^{2}\right): \alpha^{\prime} \in \mathbb{Z}[i] \text { with }\left|\alpha^{\prime}\right|=|\alpha| \text { and } w \in \mathcal{U}\right\}
$$

is a subset of $S(n, M)$. Hence we can partition $S(n, M)$ up into sets

$$
A^{*}(\alpha, \beta, u):=A(\alpha, \beta, u) \cup A(\bar{\alpha}, \bar{\beta}, \bar{u}) .
$$

How often $S(n, m)$ is equal to some unique $A^{*}(\alpha, \beta, u)$ ? We can re-formulate this question by letting

$$
\mathcal{A}(n, M):=\left\{A^{*}(\alpha, \beta, u):(\alpha, \beta, u) \in S(n, M)\right\}
$$

and asking how often $|\mathcal{A}(n, M)|>1$.

Theorem 6.2. Suppose that $M \leq 2 \sqrt{N}$ and let

$$
c=\frac{M^{2}}{4 N} \text {. }
$$

(1) The number of distinct sets $A^{*}(\alpha, \beta, u)$ with $|\alpha| \cdot|\beta| \leq \sqrt{N}$ is asymptotic to

$$
\sum_{n \leq N}|\mathcal{A}(n, M)|=\frac{\kappa^{\prime}}{2} \cdot I(c) \cdot M N^{1 / 2} \cdot\left((2 \log M)^{1 / 2}+O(1)\right) .
$$

(2) The number of pairs of distinct close-by pairs is

$$
\sum_{n \leq N}\left(\begin{array}{c}
|\mathcal{A}(n, M)| \\
2
\end{array}\right) \ll N^{1 / 3} M^{4 / 3}(\log N)^{7}+N^{1 / 2}(\log N)^{3} .
$$

Proof of Theorem 1.4 assuming Theorem 6.2. If $m$ is a non-negative integer then the characteristic function

$$
1_{\geq 1}(m)= \begin{cases}1 & m \geq 1 \\ 0 & m=0\end{cases}
$$

satisfies

$$
1_{\geq 1}(m)=m+O\left(\left(\begin{array}{l}
m \\
2
\end{array}\right)\right)
$$

and

$$
1 \geq 1(m) \leq m .
$$

Now for a given $n \in S$, and $M>0$, there exists a pair $\lambda, \lambda^{\prime} \in \mathcal{E}_{n}$ with $0<\left\|\lambda-\lambda^{\prime}\right\|<M$, if and only if $|\mathcal{A}(n, M)| \geq 1$. Hence, bearing in mind the definition (15) of $\mathcal{G}^{*}(N ; M)$, we have

$$
\mathcal{G}^{*}(N ; M)=\sum_{n \leq N} 1_{\geq 1}(|\mathcal{A}(n, M)|)
$$


Substituting (70) into (72) we obtain

$$
\begin{aligned}
\mathcal{G}^{*}(N ; M) & =\sum_{n \leq N}|\mathcal{A}(n, M)|+O\left(\sum_{n \leq N}\left(\begin{array}{c}
|\mathcal{A}(n, M)| \\
2
\end{array}\right)\right) \\
& =\frac{\kappa^{\prime}}{2} \cdot I(c) \cdot M N^{1 / 2}\left((2 \log M)^{1 / 2}+O(1)\right) \\
& +O\left(N^{1 / 3} M^{4 / 3}(\log N)^{7}+N^{1 / 2}(\log N)^{3}\right)
\end{aligned}
$$

by both parts of Theorem 6.2. The first part of Theorem 1.4 finally follows from substituting (50) into the latter estimate, recalling that here we assumed (16), and noting

$$
N^{1 / 3} M^{4 / 3}=\frac{M N^{1 / 2}}{(\sqrt{N} / M)^{1 / 3}}
$$

To prove the second part of Theorem 1.4 we substitute (71) into (72) to yield

$$
\mathcal{G}^{*}(N ; M) \leq \sum_{n \leq N}|\mathcal{A}(n, M)|=\frac{\kappa^{\prime}}{2} \cdot I(c) \cdot M N^{1 / 2}\left((2 \log M)^{1 / 2}+O(1)\right)
$$

The desired result follows at once from the fact that $I(c)$ is decreasing on $[0,1]$, so that for every $c \in[0,1], I(c) \leq I(0)=\frac{4}{\pi}$.

\subsection{Proof of Theorem 6.2, part I.}

Proof. Write $|\alpha|^{2}=a$ and $\beta=p+i q$; evidently $a \in S$. In (65), (66), (67), we see that the set $\mathcal{A}(n, M)$ is designed to take care of an automorphism group (of pairs of close-by lattice points on the circle of radius $\sqrt{n}$ ) of order 8 . Therefore

$$
\begin{aligned}
& \sum_{n \leq N}|\mathcal{A}(n, M)| \\
& \quad=\frac{1}{8} \sum_{u \in \mathcal{U}} \sum_{a \in S} \#\left\{(\beta, u) \in \mathbb{Z}[i] \times \mathcal{U}:|\beta| \leq \sqrt{N / a}, \quad|\beta-u \bar{\beta}| \leq \frac{M}{\sqrt{a}}, \quad(\beta, \bar{\beta})=1\right\},
\end{aligned}
$$

where, as before, $S$ denotes the set of integers that are sums of two squares. Let $\beta=x+i y$ so that $x+y$ is odd, and $(x, y)=1$.

In the case $u=1$ we have $|y| \leq M / 2 \sqrt{a}$ and then $x^{2} \leq N / a-y^{2}$. We will proceed analogously to the proof of Theorem 6.1, but now we have, thanks to Landau (7),

$$
S(t):=\sum_{n \in S, n \leq t} 1=\kappa_{L R} \frac{t}{(\log t)^{1 / 2}}\left(1+O\left(\frac{1}{\log t}\right)\right)
$$


so that the term on the r.h.s. of the summation in (73) corresponding to $u=1$ contributes

$$
\begin{aligned}
& \sum_{a \in S} \#\left\{(\beta, 1) \in \mathbb{Z}[i] \times \mathcal{U}:|\beta| \leq \sqrt{N / a}, \quad|\beta-\bar{\beta}| \leq \frac{M}{\sqrt{a}} \text { and }(\beta, \bar{\beta})=1\right\} \\
& =4 \sum_{y: 1 \leq y \leq M / 2} \frac{\varphi(2 y)}{2 y} \sum_{\substack{a \leq(M / 2 y)^{2} \\
a \in S}}\left(\frac{N}{a}-y^{2}\right)^{1 / 2}+O\left(\frac{M^{2}}{(\log M)^{1 / 2}}\right) \\
& =4 \sum_{y: 1 \leq y \leq M / 2-1} \frac{\varphi(2 y)}{2 y} \sum_{\substack{a \leq(M / 2 y)^{2} \\
a \in S}}\left(\frac{N}{a}-y^{2}\right)^{1 / 2}+O(N)+O\left(\frac{M^{2}}{(\log M)^{1 / 2}}\right) ;
\end{aligned}
$$

here, to avoid vanishing denominator later, we separated the contribution of $y \leq \frac{M}{2}-1$, using the trivial bound $O(N)$ to each of the summands with $y>\frac{M}{2}-1$, whose number is $O(1)$. In this case the inner sum is more complicated as compared to (56): we formally write (again hiding summation by parts followed by integration by parts in "opposite direction", much in the spirit of (56))

$$
\sum_{\substack{a \leq(M / 2 y)^{2} \\ a \in S}}\left(\frac{N}{a}-y^{2}\right)^{1 / 2}=\int_{1}^{(M / 2 y)^{2}}\left(\frac{N}{t}-y^{2}\right)^{1 / 2} d S(t)
$$

via (74) to yield

$$
\begin{aligned}
& \sum_{\substack{a \leq(M / 2 y)^{2} \\
a \in S}}\left(\frac{N}{a}-y^{2}\right)^{1 / 2} \\
& \quad=\kappa_{L R} \int_{1}^{(M / 2 y)^{2}}\left(\frac{N}{t}-y^{2}\right)^{1 / 2} \frac{d t}{(\log t)^{1 / 2}}+O\left(\frac{M N^{1 / 2}}{y(\log M / 2 y)^{3 / 2}}\right) \\
& \quad=\frac{\kappa_{L R} M N^{1 / 2}}{y(2 \log (M / 2 y))^{1 / 2}} \int_{2 y / M}^{1}\left(1-\frac{M^{2}}{4 N} v^{2}\right)^{1 / 2} d v+O\left(\frac{M N^{1 / 2}}{y(\log M / 2 y)^{3 / 2}}\right),
\end{aligned}
$$

and letting $t=(M v / 2 y)^{2}$. Extending the range of the latter integral to 0 , we see that it equals

$$
\int_{2 y / M}^{1}\left(1-\frac{M^{2}}{4 N} v^{2}\right)^{1 / 2} d v=\frac{\pi}{4} I(c)+O\left(\frac{y}{M}\right),
$$

by the definition (49) of $I(c),(68)$, and the boundedness of the integrand. We then have upon substituting the latter estimate into (76), and then into (75), that the $u=1$ term in (73) contributes to the sum

$$
\begin{aligned}
& \sum_{a \in S} \#\left\{(\beta, 1) \in \mathbb{Z}[i] \times \mathcal{U}:|\beta| \leq \sqrt{N / a}, \quad|\beta-\bar{\beta}| \leq \frac{M}{\sqrt{a}} \text { and }(\beta, \bar{\beta})=1\right\} \\
& =\pi \kappa_{L R} M N^{1 / 2} \cdot I(c) \sum_{y: 1 \leq y \leq M / 2-1} \frac{\varphi(2 y)}{2 y^{2}} \frac{1}{(2 \log (M / 2 y))^{1 / 2}}+E,
\end{aligned}
$$


where the error term $E=E(N, M)$ is bounded by

$$
\begin{aligned}
|E| & \ll \sum_{y: 1 \leq y \leq M / 2} \frac{\varphi(2 y)}{2 y}\left(\frac{M N^{1 / 2}}{y(\log M / 2 y)^{3 / 2}}+\frac{N^{1 / 2}}{(\log (M / 2 y))^{1 / 2}}\right)+\frac{M^{2}}{(\log M)^{1 / 2}} \\
& \ll M N^{1 / 2} .
\end{aligned}
$$

To evaluate the main term we reuse our estimate

$$
P(t):=\sum_{y \leq t} \frac{\varphi(2 y)}{2 y^{2}}=\frac{4}{\pi^{2}}\left(\log t+C+O\left(\frac{\log t}{t}\right)\right)
$$

for some constant $C$ (cf. (63)), and plan to use summation by parts followed by integration by parts, again in the spirit of (56). And so we get, formally manipulating (assume for simplicity that $M / 2 \in \mathbb{Z}$, otherwise further restrict the range of integration),

$$
\begin{aligned}
\sum_{y: 1 \leq y \leq M / 2-1} \frac{\varphi(2 y)}{2 y^{2}} \frac{1}{(2 \log (M / 2 y))^{1 / 2}} & =\int_{1}^{M / 2-1} \frac{d P(y)}{(2 \log (M / 2 y))^{1 / 2}} \\
& =\frac{4}{\pi^{2}} \int_{1}^{M / 2-1} \frac{d(\log y+C+O((\log y) / y))}{(2 \log (M / 2 y))^{1 / 2}} \\
& =\frac{4}{\pi^{2}} \int_{1}^{M / 2-1} \frac{d y}{y(2 \log (M / 2 y))^{1 / 2}}+E^{\prime}, \quad(79)
\end{aligned}
$$

where the error term is

$$
\left|E^{\prime}\right| \leq\left.\frac{\log y}{y(\log (M / 2 y))^{1 / 2}}\right|_{y=1} ^{y=M / 2-1}+\int_{1}^{M / 2-1} \frac{\log y d y}{y^{2}(\log (M / 2 y))^{3 / 2}} \ll \frac{\log M}{M^{1 / 2}}+1,
$$

by changing the variables $t=\frac{M}{2 y}$ and separating the contribution of the range $y \in$ $[1, \epsilon M]$ and $[\epsilon M, M / 2]$.

We may then evaluate the latter integral in (79) explicitly to be

$$
\int_{1}^{M / 2-1} \frac{d y}{y(2 \log (M / 2 y))^{1 / 2}}=(2 \log (M))^{1 / 2}+O(1)
$$

and, with the help of (80), obtain

$$
\sum_{y: 1 \leq y \leq M / 2-1} \frac{\varphi(2 y)}{2 y^{2}} \frac{1}{(2 \log (M / 2 y))^{1 / 2}}=\frac{4}{\pi^{2}}(2 \log M+O(1))^{1 / 2} .
$$


Substituting the latter estimate into (77) and bearing in mind (78) we finally obtain

$$
\begin{aligned}
& \sum_{a \in S} \#\left\{(\beta, 1) \in \mathbb{Z}[i] \times \mathcal{U}:|\beta| \leq \sqrt{N / a}, \quad|\beta-\bar{\beta}| \leq \frac{M}{\sqrt{a}} \text { and }(\beta, \bar{\beta})=1\right\} \\
& =\frac{4}{\pi} \kappa_{L R} M N^{1 / 2} \cdot I(c) \cdot(2 \log M+O(1))^{1 / 2},
\end{aligned}
$$

which contributes a quarter in (73) of what is stated in part I of Theorem 6.2. We get a similar quantity for $u=-1$; and by suitably modifying the proof, we get the same quantity for $u=i$ and $u=-i$, modifying the proof much like we did in Theorem 6.1.

\subsection{Many pairs-proof of Theorem 6.2, part II.}

Lemma 6.3. For any $\theta$ we have, uniformly,

$\#\{x+i y \in \mathbb{Z}[i]:|x+i y| \leq N,(x, y)=1 \&|\arg (x+i y)-\theta|<\epsilon\} \ll 1+\epsilon N^{2}$.

In fact if $\epsilon \leq 1 / 2 N^{2}$ there is no more than one solution.

The proof of Lemma 6.3 will be given immediately after the proof of Theorem 6.2 , part II.

Proof of Theorem 6.2, part II assuming Lemma 6.3. We treat separately those $n \asymp N$, which are either a square nor twice a square. These contribute at most

$$
\sum_{m^{2} \asymp N} r_{2}\left(m^{2}\right)^{2}+\sum_{2 m^{2} \asymp N} r_{2}\left(2 m^{2}\right)^{2} \ll N^{1 / 2}(\log N)^{3} .
$$

Now suppose we have two pairs $a_{1}, b_{1}$ and $a_{2}, b_{2}$ not belonging to the same class $A^{*}(\alpha, \beta, u)$, but with the same $n \asymp N$, which is neither a square nor twice a square. We use the identification between the close-by pairs and triples $(\alpha, \beta, u) \in S(n, M)$ as in the beginning of section 6.1, so that a couple of close-by pairs yields two triples $\left(\alpha_{j}, \beta_{j}, u_{j}\right) \in S(n, M), j=1,2$. It is then possible to write

$$
\beta_{1}=\gamma \delta \theta_{1} \text { and } \beta_{2}=\gamma \bar{\delta} \theta_{2}
$$

where $\left(\beta_{1}, \beta_{2}\right)=(\gamma)$ and $\left(\beta_{1} / \gamma, \overline{\beta_{2} / \gamma}\right)=(\delta)$, and the norms of $\theta_{1}$ and $\theta_{2}$ are coprime. Since $\left|\alpha_{1} \beta_{1}\right|=\left|\alpha_{2} \beta_{2}\right|=\sqrt{n}$, we have

$$
\alpha_{1}=t_{2} v_{1} \text { and } \alpha_{2}=t_{1} v_{2},
$$

where

$$
\left|t_{1}\right|=\left|\theta_{1}\right|,\left|t_{2}\right|=\left|\theta_{2}\right| \text { and }\left|v_{1}\right|=\left|v_{2}\right| \text {. }
$$

Hence we must have

$$
\left|\gamma \delta \theta_{1} \theta_{2} v_{1}\right|=\left|\beta_{1} \alpha_{1}\right|=\sqrt{n} \asymp \sqrt{N},
$$

and we in addition have

$$
\left|\theta_{1} v_{1}\right|=\left|\alpha_{2}\right|=\frac{M}{\left|\beta_{2}-u \overline{\beta_{2}}\right|} \leq M, \text { and }\left|\theta_{2} v_{1}\right|=\left|\alpha_{1}\right| \leq M
$$


in a similar fashion. Substituting the estimates (86) into (85) we conclude that

$$
\left|\gamma \delta \theta_{1}\right|,\left|\gamma \delta \theta_{2}\right| \gg \frac{\sqrt{N}}{M} .
$$

Next we use the condition that

$$
\left|\alpha_{j}\right| \cdot\left|\beta_{j}-u_{j} \overline{\beta_{j}}\right| \leq M .
$$

Since $\left|\alpha_{j}\right| \cdot\left|\beta_{j}\right|=\sqrt{n} \asymp \sqrt{N}$, this yields

$$
\left|1-u_{j} \cdot \exp \left(-2 i \cdot \arg \left(\beta_{j}\right)\right)\right|=\left|1-u_{j} \frac{\overline{\beta_{j}}}{\beta_{j}}\right| \ll \frac{M}{\sqrt{N}} .
$$

Recalling that $u_{j}$ are units, this implies that $2 \arg \left(\beta_{j}\right)$ are small modulo $\frac{\pi}{2}$, or, more precisely

$$
\left|\arg \left(\beta_{j}\right)\right| \ll \frac{M}{\sqrt{N}} \bmod \frac{\pi}{4} .
$$

Therefore, bearing in mind (82), we have

$$
\begin{array}{lll}
\arg \left(\theta_{1}\right)=-\arg \gamma-\arg \delta & (\bmod \pi / 4) & +O(M / \sqrt{N}) \\
\arg \left(\theta_{2}\right)=-\arg \gamma+\arg \delta & (\bmod \pi / 4)+O(M / \sqrt{N})
\end{array}
$$

We have two linearly independent equations in four unknowns. This allows us to determine $\arg \gamma, \arg \delta$ in terms of $\arg \left(\theta_{1}\right), \arg \left(\theta_{2}\right) \bmod \pi / 4$, with error $O(M / \sqrt{N})$ :

$$
\begin{array}{ll}
2 \arg \gamma+\left(\arg \left(\theta_{1}\right)+\arg \left(\theta_{2}\right)\right) & (\bmod \pi / 4)=O(M / \sqrt{N}) \\
2 \arg \delta+\left(\arg \left(\theta_{1}\right)-\arg \left(\theta_{2}\right)\right) & (\bmod \pi / 4)=O(M / \sqrt{N}) .
\end{array}
$$

The inequalities (88) imply that there exist $w, w^{\prime} \in \pm\{1, i, 1+i, 1-i\}$ such that

$$
\arg \left(w \delta^{2} \theta_{1} \overline{\theta_{2}}\right), \arg \left(w^{\prime} \gamma^{2} \theta_{1} \theta_{2}\right)=O\left(\frac{M}{\sqrt{N}}\right) .
$$

We argue that neither of the numbers on the 1.h.s. of (89) can vanish precisely: For if we suppose that $\arg \left(w \delta^{2} \theta_{1} \overline{\theta_{2}}\right)=0$, then, as $\theta_{1}$ and $\theta_{2}$ are coprime, we can write $\theta_{j}=r_{j} \phi_{j}^{2} \omega_{j}$ for $j=1,2$ with $\delta=\overline{\phi_{1}} \phi_{2}$, where each $\omega_{i}$ divides $\omega$, and the $r_{j}$ are integers. Now $\left(r_{j} \omega_{j}\right)$ divides $\left(\theta_{j}, \overline{\theta_{j}}\right)$, which divides $\left(\beta_{j}, \overline{\beta_{j}}\right)=1$, and so $r_{j} \omega_{j}$ is a unit. Moreover

$$
\phi_{1} \phi_{2} \mid\left(\delta \theta_{1}, \bar{\delta} \theta_{2}\right)=\left(\beta_{1} / \gamma, \beta_{2} / \gamma\right)=(1),
$$

and so $\phi_{1}=\phi_{2}=1$, and therefore $\delta=\overline{\phi_{1}} \phi_{2}=1$. Therefore $\theta_{1}, \theta_{2}$ are units. We may then select $\gamma$ so that $\theta_{2}=1$, and so $\beta_{2}=w \beta_{1}$ for some unit $w$, and $\left|\alpha_{1}\right|=\left|\alpha_{2}\right|$. Therefore $\left(\alpha_{2}, \beta_{2}, u_{2}\right) \in A\left(\alpha_{1}, \beta_{1}, u_{1}\right)$ in contradiction to our assumption that these triples belong to different classes $A^{*}(\alpha, \beta, u)$. Similarly if $\arg \left(w^{\prime} \gamma^{2} \theta_{1} \theta_{2}\right)=0$ then we take the conjugate of $\left(\alpha_{2}, \beta_{2}\right)$, so that the roles of $\gamma$ and $\delta$ are exchanged, and so $\left(\alpha_{2}, \beta_{2}, u_{2}\right) \in A\left(\overline{\alpha_{1}}, \overline{\beta_{1}}, \overline{u_{1}}\right)$. In either case we get a contradiction to our assumption that $\left(\alpha_{j}, \beta_{j}, u_{j}\right), j=1,2$ belong to different classes $A^{*}(\alpha, \beta, u)$. 
Therefore we may assume

$$
0 \neq \arg \left(w \delta^{2} \theta_{1} \overline{\theta_{2}}\right)=O\left(\frac{M}{\sqrt{N}}\right)
$$

and so

$$
1 \leq\left|\operatorname{Im}\left(w \delta^{2} \theta_{1} \overline{\theta_{2}}\right)\right| \asymp\left|\arg \left(w \delta^{2} \theta_{1} \overline{\theta_{2}}\right)\right| \cdot\left|\delta^{2} \theta_{1} \overline{\theta_{2}}\right| \ll\left|\delta^{2} \theta_{1} \overline{\theta_{2}}\right| \cdot \frac{M}{\sqrt{N}},
$$

and so we deduce that

$$
\left|\gamma^{2} \theta_{1} \theta_{2}\right| \gg \frac{\sqrt{N}}{M}, \text { and }\left|\delta^{2} \theta_{1} \overline{\theta_{2}}\right| \gg \frac{\sqrt{N}}{M} .
$$

To summarize all the above, a pair of triples $\left(\alpha_{j}, \beta_{j}, u_{j}\right)$ that satisfy the conditions above and belong to different classes $A^{*}(\cdot, \cdot, \cdot)$ determines an 8 -tuple $\left(\gamma, \delta, \theta_{1}, \theta_{2}, t_{1}, t_{2}\right.$, $\left.v_{1}, v_{2}\right) \in \mathbb{Z}[i]^{8}$ that satisfies (84), (85), (86), (87) and (90). Conversely, such a 8 tuple corresponds to a unique $\left(\alpha_{j}, \beta_{j}\right)$ via (82) and (83), hence, for our purposes it is sufficient to bound their number (bearing in mind that the group of units is finite). Let $C, D, T_{1}, T_{2}, V$ be powers of 2 that are greater than and closest to $|\gamma|,|\delta|,\left|\theta_{1}\right|=$ $\left|t_{1}\right|,\left|\theta_{2}\right|=\left|t_{2}\right|$, and $\left|v_{1}\right|=\left|v_{2}\right|$ respectively; hence their product is $C D T_{1} T_{2} V \asymp \sqrt{N}$ with $T_{1} V, T_{2} V \ll M$, and

$$
C D T_{1}, C D T_{2}, C^{2} T_{1} T_{2}, D^{2} T_{1} T_{2} \gg \sqrt{N} / M
$$

We note that $\sum_{n \leq N} r_{2}(n)^{2} \asymp N \log N$. First select an integer $v \asymp V^{2}$ and any $v_{1}, v_{2}$ with $\left|v_{1}\right|^{2}=\left|v_{2}\right|^{2}=v$, so the total number of possible choices for $v_{1}, v_{2}$ is

$$
\leq \sum_{v \ll V^{2}} r_{2}(v)^{2} \ll V^{2} \log V \ll V^{2} \log M
$$

Let $A$ be the largest of $C, D, T_{1}, T_{2}$, and $B$ be the second largest. By (91), we have

$$
A B \gg\left(\frac{\sqrt{N}}{M}\right)^{2 / 3} \text { and } B \gg\left(\frac{\sqrt{N}}{M}\right)^{1 / 4},
$$

the former following from the fact that the product $A B$ must appear in either of $C D T_{1}$, $C D T_{2}$, and the latter is a consequence of the fact that $B$ must appear in one of the 4 products on the 1.h.s. of (91) without $A$. If the two largest are, say, $C$ and $D$, then we select any $\theta_{1}, t_{1}$ with $\left|\theta_{1}\right|^{2}=\left|t_{1}\right|^{2}=r \asymp T_{1}^{2}$, and $\theta_{2}, t_{2}$ with $\left|\theta_{2}\right|^{2}=\left|t_{2}\right|^{2}=s \asymp T_{2}^{2}$, so the number of choices of these are

$$
\ll T_{1}^{2} \log T_{1} \cdot T_{2}^{2} \log T_{2} \ll\left(T_{1} T_{2} \log M\right)^{2},
$$

similar to (92). Next we select $\gamma$ with $|\gamma| \asymp C$, and $\delta$ with $|\delta| \asymp D$, with their arguments in the narrow intervals, of width $O(M / \sqrt{N})$, given by the equations (88) above. Then, by Lemma 6.3, the number of such $\gamma$ is $\ll 1+C^{2} \frac{M}{\sqrt{N}}$, and the number of such $\delta$ is $\ll 1+D^{2} \frac{M}{\sqrt{N}}$. Hence, the ordering assumptions on $C, D, T_{1}, T_{2}, V$ above, the total 
number of possible $\left(\gamma, \delta, \theta_{1}, \theta_{2}, t_{1}, t_{2}, v_{1}, v_{2}\right)$ (and hence the corresponding $\left(\alpha_{j}, \beta_{j}\right)$, $j=1,2)$ is

$$
\begin{aligned}
\ll & \left(V^{2} \log M\right) \cdot\left(T_{1} T_{2} \log M\right)^{2} \cdot\left(1+C^{2} \frac{M}{\sqrt{N}}\right) \cdot\left(1+D^{2} \frac{M}{\sqrt{N}}\right) \\
& =\left(C D T_{1} T_{2} V\right)^{2}(\log M)^{3} \cdot\left(\frac{1}{C^{2}}+\frac{M}{\sqrt{N}}\right) \cdot\left(\frac{1}{D^{2}}+\frac{M}{\sqrt{N}}\right) \\
& \ll N\left(\frac{1}{A^{2}}+\frac{M}{\sqrt{N}}\right) \cdot\left(\frac{1}{B^{2}}+\frac{M}{\sqrt{N}}\right) \cdot(\log M)^{3},
\end{aligned}
$$

and the analogous expression is proved for every ordering of $C, D, T_{1}, T_{2}$. Thanks to (93), each of these expressions is $\ll N^{1 / 3} M^{4 / 3}(\log N)^{3}$, and, since the numbers $C, D, T_{1}, T_{2}$, whose product is $\ll N$, are all powers of 2 , the number of possibilities for choosing them is $\ll(\log N)^{4}$, which implies (cf. (81))

$$
\sum_{n \leq N}\left(\begin{array}{c}
|\mathcal{A}(n, M)| \\
2
\end{array}\right) \ll N^{1 / 3} M^{4 / 3}(\log N)^{7}+N^{1 / 2}(\log N)^{3},
$$

claimed in (69).

Remark 6.1. There are $\asymp M^{2}(\log M)^{7}$ solutions with $C, D, T_{1}, T_{2} \gg(\sqrt{N} / M)^{1 / 2}$, and we expect this to be the correct number of solutions, provided that $M>N^{c}$ for some $c>0$.

Proof of Lemma 6.3. Multiplying through by the units we can place $\theta$ in the first quadrant; swapping $x$ and $y$ if necessary we may assume that $0<y<x<N$. Since the derivative of arctan is bounded away from 0 , our set cardinality is bounded by

$$
\begin{aligned}
\#\{x+i y \in \mathbb{Z}[i]:|x+i y| \leq N, & (x, y)=1 \&|\arg (x+i y)-\theta|<\epsilon\} \\
& \ll \#\{0<y<x<N:|y / x-\theta|<\epsilon\} .
\end{aligned}
$$

If there is no solution to (94) we are done, otherwise we choose one, i.e. a tuple $(a, b) \in \mathbb{Z}^{2}$ with $0<b<a<N$ and $(a, b)=1$ such that $|b / a-\theta|<\epsilon$. Any other solution $(x, y)$ of $(94)$ satisfies

$$
\frac{1}{N^{2}}<\frac{1}{x b} \leq\left|\frac{y}{x}-\frac{a}{b}\right| \leq\left|\frac{y}{x}-\theta\right|+\left|\theta-\frac{a}{b}\right|<2 \epsilon,
$$

hence there is at most one solution unless $\epsilon>1 / 2 N^{2}$. The above implies that every other solution $(x, y)$ of (94) necessarily satisfies

$$
|a x-b y|<2 \epsilon b x .
$$

Now, since $(a, b)=1$, there exists an integer solution $(u, v)=\left(u_{0}, v_{0}\right)$ to $a u-b v=$ 1 , and hence for $d \in \mathbb{Z}$ the tuple $(x, y)=\left(d u_{0}, d v_{0}\right)$ is one solution to

$$
a x-b y=d \text {. }
$$

Hence the general solution to (96) is given by $x=d u_{0}+k b, y=d v_{0}+k a$ with $k$ integer; a solution of (95) is a solution to (96) with $|d|<2 \epsilon b x \ll \epsilon b N$, and, given a value of $d$, the condition $0<y=d v_{0}+k a<N$ forces $k$ to lie in an interval of length $O(N / a)$. Therefore the total number of solutions to (95) is

$$
\ll \epsilon b N \cdot \frac{N}{a} \ll \epsilon N^{2},
$$

which implies the statement of Lemma 6.3. 


\section{Best Possible?}

7.1. Squarefree $n$ : best possible?. It is believed that there are lots of primes of the form $a^{2}+1$. Say $p_{1}, \ldots, p_{k}$ are primes, close to $y^{2}$, with $p_{j}=a_{j}^{2}+1$ so each $a_{j}$ is very close to $y$.

Then $P_{j}=a_{j}+i$, and these are each complex numbers with $\left|\arg \left(a_{j} \pm i\right)\right| \ll 1 / y$. Therefore any product $\left(a_{1} \pm i\right) \cdots .\left(a_{k} \pm i\right)$ has argument $\ll k / y$. Therefore these points appear in four arcs of width $\ll k / y$ centered around $1, i,-1,-i$. Here $n=p_{1} \ldots p_{k} \approx$ $y^{2 k}$ and so the width of these arcs is $\ll k / n^{1 / 2 k}$. This yields examples of $n$ with $n$ is squarefree and $r_{2}(n) \rightarrow \infty$ for which

$$
\#\left\{\alpha, \beta \in \mathcal{E}_{n}:|\alpha-\beta| \leq n^{1 / 2-o(1)}\right\} \gg\left|\mathcal{E}_{n}\right|^{2},
$$

where $o(1)$ is going to 0 arbitrarily slowly.

7.2. Very short arcs. Fix an integer $a$ composed of many prime factors $\equiv 1(\bmod 4)$, and select $m$ arbitrarily large, for which $b=m^{2}+1$ has $\leq 3$ prime factors. ${ }^{2}$ Let $u=1$. If $|\alpha|^{2}=a$ then $A^{*}(\alpha, m+i, 1) \subset S\left(a b, 2 a^{1 / 2}\right)$, which embeds into $D\left(a b, 2 / b^{1 / 2}\right) \subset$ $D(a b, 2 / m)$. Now $\left|A^{*}(\alpha, m+i, 1)\right| \gg r_{2}(a) \gg|D(a b)|$. Therefore, for $n=a b$, we have given infinitely many examples with

$$
\#\left\{(u, v) \in \mathcal{E}_{n}:|u-v| \leq n^{o(1)}\right\} \gg\left|\mathcal{E}_{n}\right| .
$$

Acknowledgements. The authors would like to thank Mike Bennett, Valentin Blomer, Jerry Buckley, Stephen Lester, Zeév Rudnick, Mikhail Sodin, and Peter Sarnak for a number of stimulating and fruitful conversations and their remarks. It is a pleasure to thank the anonymous referee, whose numerous comments have helped us improve the presentation of the results. The research leading to these results has received funding from the European Research Council under the European Union's Seventh Framework Programme (FP7/2007-2013), ERC grant agreement $n^{\circ} 670239$ (A.G.) and $n^{\circ} 335141$ (I.W.), as well as from NSERC Canada under the CRC program (A.G.).

Open Access This article is distributed under the terms of the Creative Commons Attribution 4.0 International License (http://creativecommons.org/licenses/by/4.0/), which permits unrestricted use, distribution, and reproduction in any medium, provided you give appropriate credit to the original author(s) and the source, provide a link to the Creative Commons license, and indicate if changes were made.

\section{References}

1. Berry, M.: Regular and irregular semiclassical wavefunctions. J. Phys. A. 10(12), 2083-2091 (1997)

2. Berry, M.: Semiclassical mechanics of regular and irregular motion. Chaotic behavior of deterministic systems (Les Houches, 1981), pp. 171-271, North-Holland, Amsterdam (1983)

3. Bourgain, J., Rudnick, Z.: On the geometry of the nodal lines of eigenfunctions of the two-dimensional torus. Ann. Henri Poincaré. 12(6), 1027-1053 (2011)

4. Brüdern, J.: Einführung in die analytische Zahlentheorie. Springer, Berlin (1995)

5. Cilleruelo, J., Cordoba, A.: Trigonometric polynomials and lattice points. Proc. Am. Math. Soc. 115(4), 899-905 (1992)

6. Cilleruelo, J., Granville, A.: Lattice points on circles, squares in arithmetic progressions, and sumsets of squares. In: Additive Combinatorics, CRM Proceedings \& Lecture Notes, Vol. 43, pp. 241-262 (2007)

7. Colin de Verdière, Y.: Ergodicité et fonctions propres du Laplacien. Commun. Math. Phys. 102, 497502 (1985)

8. Granville, A., Stark, H.M.: ABC implies no "Siegel Zeroes" for L-functions of characters with negative discriminant. Invent. Math. 139, 509-523 (2000)

\footnotetext{
${ }^{2}$ We know such $b$ exist by sieve methods; though we believe there are infinitely many such primes, which is as yet unproved.
} 
9. Han, X.: Small scale quantum ergodicity in negatively curved manifolds. Nonlinearity 28(9), 3263$3288(2015)$

10. Han, X.: Small scale equidistribution of random eigenbases. Commun. Math. Phys. 349(1), 425440 (2017)

11. Hezari, H., Rivière, G.: $L^{p}$ norms, nodal sets, and quantum ergodicity. Adv. Math. 290, 938-966 (2016)

12. Hezari, H., Rivière, G.: Quantitative equidistribution properties of toral eigenfunctions. J. Spectr. Theory 7(2), 471-485 (2017)

13. Landau, E.: Über die Einteilung der positiven ganzen Zahlen in vier Klassen nach der Mindeszahl der zu ihrer additiven Zusammensetzung erforderlichen Quadrate. Arch. Math. Phys. 13, 305-312 (1908)

14. Lester, S., Rudnick, Z.: Small scale equidistribution of eigenfunctions on the torus. Commun. Math. Phys. 350(1), 279-300 (2017)

15. Luo Zhi, W., Sarnak, P.: Quantum ergodicity of eigenfunctions on $P S L_{2}(\mathbb{Z}) \backslash \mathbb{H}^{2}$. Inst. Hautes Tudes Sci. Publ. Math. No. 81, 207-237 (1995)

16. Snirel'man, A.: Ergodic properties of eigenfunctions. Uspekhi Mat. Nauk 180, 181-182 (1974)

17. Young, M.: The quantum unique ergodicity conjecture for thin sets. Adv. Math. 286, 958-1016 (2016)

18. Zelditch, S.: Uniform distribution of eigenfunctions on compact hyperbolic surfaces. Duke Math. J. 55, 919-941 (1987)

Communicated by J. Marklof 